\title{
Testing a community-based distribution approach to reproductive health service delivery in Senegal (a study of community agents in Kébémer)
}

Diouratie Sanogo

Mady Cisse

Adama Ndoye

Laty G. Ndoye

Ousmane Faye

See next page for additional authors

Follow this and additional works at: https://knowledgecommons.popcouncil.org/departments_sbsr-rh

Part of the Family, Life Course, and Society Commons, International Public Health Commons, and the Maternal and Child Health Commons

How does access to this work benefit you? Let us know!

\section{Recommended Citation}

Sanogo, Diouratie, Mady Cisse, Adama Ndoye, Laty G. Ndoye, Ousmane Faye, and Balla Mbacke Mboup. 2004. "Testing a community-based distribution approach to reproductive health service delivery in Senegal (a study of community agents in Kébémer)," FRONTIERS Final Report. Washington, DC: Population Council. 
Authors

Diouratie Sanogo, Mady Cisse, Adama Ndoye, Laty G. Ndoye, Ousmane Faye, and Balla Mbacke Mboup 


\title{
Testing a Community Based Distribution Approach to Reproductive Health Service Delivery in Senegal (a study of community agents in Kébémer)
}

\author{
Diouratié SANOGO, Mady CISSE \\ Médecin-Colonel Adama NDOYE, Laty G. NDOYE \\ Ousmane Faye, Balla Mbacké MBOUP \\ Population Council \\ Ministry of Health, Division of Reproductive Health (DSR) \\ Management Sciences for Health (MSH) \\ Health District of Kébémer
}

March 2004

This study was funded by the U.S. AGENCY FOR INTERNATIONAL DEVELOPMENT (USAID) under the terms of the Frontiers in Reproductive Health Program Copoerative Agreement No. HRN-A-00-98-00012-00 and Population Council in-house project no. 8010 13049. The opinions expressed herein are those of the authors and do not necessarily reflect the views of USAID. 


\section{SUMMARY}

The Division of Reproductive Health (DSR) of the Senegal Ministry of Health and Social Action, in partnership with the Population Council's FRONTIERS in Reproductive Health program and Management Sciences for Health (MSH), conducted a study to test and compare three ways of providing reproductive health services to rural communities in the Kébémer district in terms of their effectiveness, cost and cost-effectiveness. FRONTIERS and MSH collaborated with the DSR to design the interventions, MSH supported the DSR to implement the interventions, and FRONTIERS undertook the evaluation. USAID provided funding for the study.

This study responded to the recommendations of a 1999 workshop, organized by FRONTIERS and the DSR, on the community-based distribution (CBD) approach, which defined alternative CBD models appropriate for Senegal. The DSR sees the development of community-based service delivery models as essential for the future of health care in Senegal.

\section{Objectives}

The general objective of this study was to contribute to the development of an integrated cost-effective program to increase the accessibility and availability of reproductive health information and services in rural areas of Senegal. Specifically, the study sought to:

- Determine the acceptability and feasibility of two alternative community-based service delivery models.

- Compare the effectiveness of these two models with that of a clinic-strengthening model in terms of knowledge and utilization of basic reproductive health services by rural women.

- Compare the performance of community-level workers in each model.

- Estimate the costs and cost-effectiveness of each model.

- Draw lessons and make recommendations for introducing a cost-effective community based reproductive health services public sector model for Senegal.

\section{Description of Study Interventions}

The interventions consisted of the following components:

\section{Development and introduction of two CBD models in Kébémer district:}

- One model used the Agents de Santé Communautaires (ASC), who currently work in 'community health huts'. In 15 functioning health huts, additional supplies and equipment were provided following a needs assessment. Two ASC were selected at each of the 15 health huts, and the 30 ASCs were given a two-week training course to become CBD agents.

- The other model created a new cadre of community-based workers termed Relais Communautaires (RC). These were used where a health hut existed but was not currently functioning. Two RCs (a man and a woman) were selected using criteria developed at the 1999 workshop: literate; long-term community resident; married; aged 20-45 years; recognized and trusted by the community; demonstrated attitude of service to the community; and having the confidence of the community. The $30 \mathrm{RCs}$ received the same two-week training as the ASCs. 
Over a twelve-month intervention period, the $30 \mathrm{ASCs}$ and the $30 \mathrm{RCs}$ were expected to: conduct home visits; hold talks on reproductive health issues and services in their respective communities; sell reproductive health commodities; and make referrals to the health posts for other services. Four reproductive health services were provided:

- Family planning: information on contraceptive methods, selling condoms and spermicides, and referrals for clinical contraceptives.

- STIs and HIV/AIDS: information on symptoms and means of prevention, and referrals for treatment.

- Safe motherhood: information on antenatal care, referral for antenatal care services, follow-up of pregnant women, and selling vitamin A and iron tablets.

- Child survival: information on practices harmful to child survival; referrals and follow-up for infant immunization and growth monitoring; information on malaria; and selling chloroquine, ORS, and vitamin A.

\section{Strengthening services at the clinic level}

At the Health District level, the ten nurses at the ten Health Posts received a twelve-day training on reproductive health issues to enable them to become trainers and supervisors of the ASCs and RCs in their respective areas. Their training was based on four modules: the CBD approach in the context of the Senegal reproductive health policy; the components of community health services; information, education and counseling on reproductive health issues; and management of community health services.

\section{Evaluation design}

The study developed, tested and compared the following three service delivery models:

- Strengthening the health posts only.

- Strengthening the functional capacity of 15 community health huts and their ASCs to offer a range of reproductive health services in addition to strengthening.

- Introducing a new cadre of community-based workers termed Relais Communautaires (RC) to work in the non-functioning health huts.

The three service delivery models were tested within the district of Kébémer to maintain similarity in situational characteristics. The district was divided into three geographicaladministrative zones on the basis of the locations of the ten Health Posts; thus one zone had four contiguous Health Posts and the other two had three contiguous Health Posts. There is only one District Health Center, and to control for its possible effect on the two communitybased models, the zone in which the District Health Center is located was assigned to be the site for testing the clinic-strengthening model. The two community-based models were then randomly assigned to the other two zones. Kébémer district, located about 160 kilometers north of Dakar, has about 112,000 inhabitants and about 600 villages. The two zones to which the community-based interventions were allocated each have three Health Posts and approximately 24 Health Huts, of which approximately 15 were functional. Each Health Post serves approximately 60 villages, and so each Health Hut serves approximately eight villages and a total population of 1,500 persons, or 200-300 households.

Data for designing and evaluating the interventions were collected through a series of quantitative and qualitative studies including: 
- A baseline and follow-up survey after 12 months of implementing the interventions to measure population-level knowledge of reproductive health issues, and their perceptions and use of reproductive health services;

- An assessment of functioning of all health facilities in the health district, including monthly service statistics to measure the performance of the clinics and CBD agents;

- Focus group discussions and in-depth interviews after 12 months of implementation with key informants to describe perceptions of the implementation processes; and

- A cost analysis to determine the costs and cost-effectiveness of each intervention.

\section{Results}

Qualitative data show that health care providers and community leaders found both models acceptable and are ready to adopt them. For the DSR, the pictorial instruments developed for the CBD agent activity reports, the CBD agent training modules, and the experience gained from participation in the OR process were the most important results of the project. For women and men in the communities, the ASC model was slightly more acceptable. The preference for the ASC model may be due to their previous experience with the community and also to the fact that the ASC are almost all women. However, higher proportions of women $(64 \%-71 \%)$ than men $(46 \%$ - $48 \%)$ reported ever interacting with a CBD agent, and interaction was slightly higher for the RC model.

Statistics from the CBD agent monthly activity reports indicate that, overall, the agents were holding $3-4$ health talks, $1-2.5$ home visits and $1-2.5$ referrals per agent per month. They were also selling contraceptives to an average of less than one client per month, and other health commodities to $8-12$ clients per month. The statistics suggest that the performance of the RCs was higher than that of the ASCs. On average, the RCs served 1.35 times more contraceptive clients and 1.41 times more maternal child health clients, and made 1.39 times more referrals than the ASCs; although the ASCs made twice as many home visits as the RCs.

Although women living in villages where the two CBD models were introduced had significantly higher levels of knowledge of family planning and HIV/AIDS after introduction of the interventions, a similarly significant increase was found in the comparison site, and so the CBD models did not have an effect over and above general improvements in knowledge. A significant increase in knowledge of safe motherhood issues was found for the RC model only, and significant increases in child survival knowledge were found in both the RC and comparison sites. Significant improvements in attitudes towards family planning and towards discussion of HIV/AIDS in public venues were observed in the two CBD sites only and not in the comparison site.

Ever use of family planning by married women during the past 12 months increased after introduction of the two CBD models, but these increases were only statistically significant (at 0.05 level) for the model using the RCs (an increase from 3.3 to 4.2 percent). There was no change in the comparison site; indeed, a significant decrease in women currently using a method at the time of the survey was observed in the comparison site. These findings indicate that the CBD models may be countering a stagnation or decline in family planning use in Kébémer district. There was no change in any sites in the use of condoms by men and, as would be expected, condom use was much higher among non-married than married men. 
The cost analysis indicates that marginal costs of implementing the ASC model $(\$ 26,546)$ were much less than for the RC model $(\$ 38,512)$, primarily because of the high costs associated with transportation for the RCs (i.e. purchase and upkeep of the horse and cart). In terms of cost-effectiveness also, the ASC model proved to be more efficient than the RC model.

\begin{tabular}{|l|c|c|}
\hline \multicolumn{1}{|c|}{ Cost-effectiveness indicator } & ASC model & RC model \\
\hline Cost per family planning client supplied & $\$ 139$ & $\$ 149$ \\
\hline Cost per other medication client supplied & $\$ 10$ & $\$ 12$ \\
\hline Cost per client referred for an RH service & $\$ 57$ & $\$ 59$ \\
\hline Cost per client served & $\$ 8$ & $\$ 9$ \\
\hline
\end{tabular}

These results suggest that serving a family planning client is by far the most expensive activity for the CBD agents. The data also indicate that an increase by one percentage point in the contraceptive prevalence rate can be achieved by spending $\$ 8,858$ in the $\mathrm{RC}$ model, compared with $\$ 2,048$ in the ASC model. As there was no increase in contraceptive prevalence in the comparison site, it is not possible to compare these models with the facilitystrengthening only model.

In summary, neither model proved particularly successful in terms of increasing knowledge, attitudes and use of family planning compared with the comparison site. However, the comparison site was in a more urbanized and developed area than the two CBD sites, which were in rural, largely inaccessible areas, and levels of knowledge, attitudes and use were already much higher in the comparison area. The $\mathrm{RC}$ model, which created and introduced a new cadre of outreach community-based worker to replace non-functioning health huts, was found to be less effective and less cost-effective than the ASC model, which strengthened existing ASCs working in functioning health huts. Consequently, if functioning health huts exist, it would appear that strengthening them would be an appropriate way for the DSR to reach rural populations. In areas where there are no functioning health huts, it would seem appropriate for the DSR to consider ensuring that they can start to function again and then strengthen the ASCs.

\section{Dissemination and utilization}

A one-day seminar, attended by over 120 participants from all levels of the health care system, was organized to present the results in Kébémer. In addition, a one-day seminar was organized in each of the six rural communities where the two CBD models were tested to present and discuss their specific results. Over 60 participants attended each of these seminars. The DSR's CBD technical committee met to plan for utilization of the study findings. The major outcomes of these meetings were agreement that both CBD models are suitable ways to quickly expand access to reproductive health services, although appropriate monitoring and supervision mechanisms need to be developed to ensure quality of care.

One aspect of this project that has received considerable attention is the "community participation" process used during implementation. This process has already been adopted and used by some NGOs working in other regions of the country: AFRICARE and ASBEF in Kaolack, ACDEV in Fatick, and FANKATA in Kolda.

Following is the full version of the above-summarized report in the original French. 


\section{TABLE DES MATIERES}

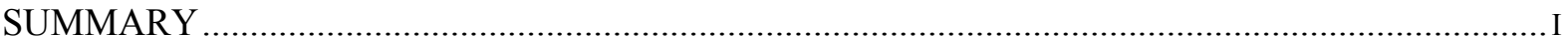

LISTE DES TABLEAUX ET DES GRAPHIQUES ...........................................................................

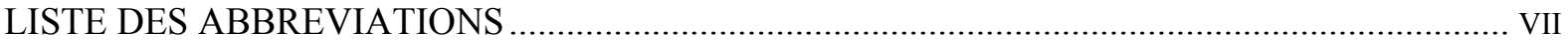

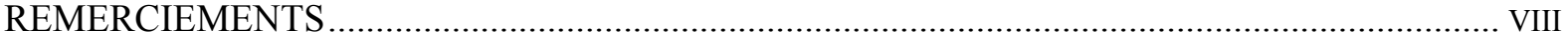

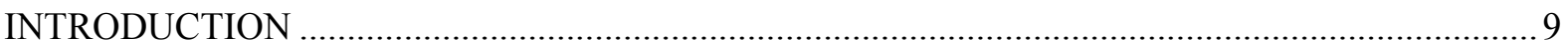

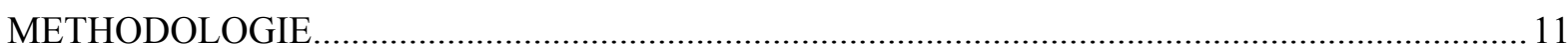

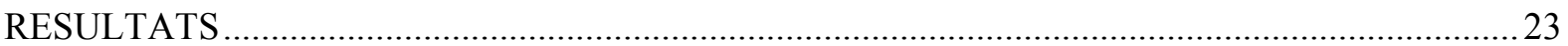

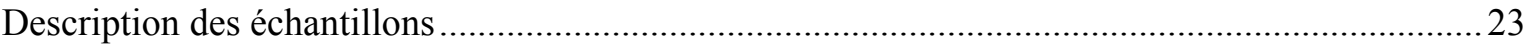

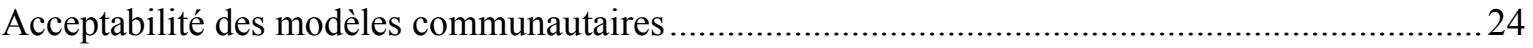

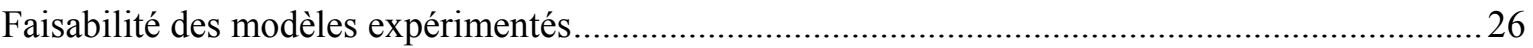

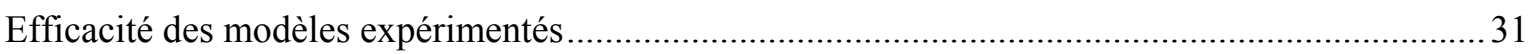

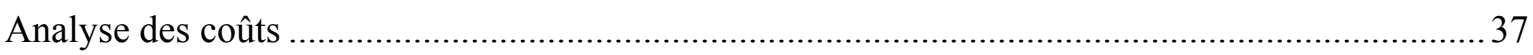

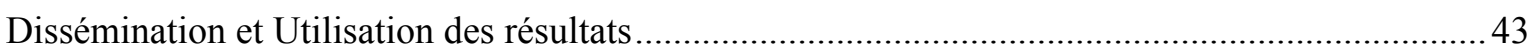

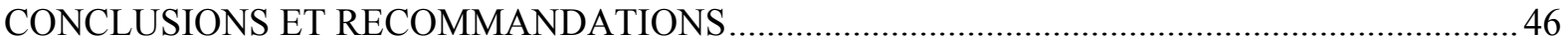

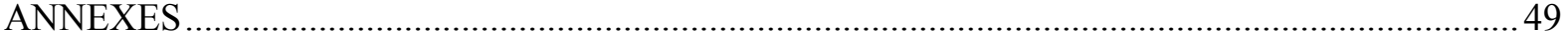




\section{LISTE DES TABLEAUX ET DES GRAPHIQUES}

\section{Tableaux}

Tableau 1 : Types de services disponibles selon le niveau de prestation et la composante de SR

Tableau 3a : Indicateurs d'acceptabilité des modèles communautaires au niveau des femmes ......... 24

Tableau 3b : Indicateurs d'acceptabilité des modèles communautaires au niveau des hommes ........ 25

Tableau 4: Nombre d'activités réalisées par tous les ASBC au cours des 10 premiers mois du projet.

Tableau 5 : Indice synthétique du niveau de connaissance en SR ........................................... 32

Tableau 6a : Coûts des activités communes aux modèles expérimentés ......................................... 40

Tableau 6b : Coûts marginaux des modèles expérimentés ........................................................... 41

Tableau 7a : Rentabilité du modèle communautaire avec ASC ......................................................42

Tableau 7b : Rentabilité du modèle communautaire avec RC ......................................................42

\section{Tableaux Annexes}

Tableau 2a : Caractéristiques socio-démographiques des femmes selon la zone d'étude.......38

Tableau 2b : Caractéristiques socio-démographiques des hommes selon la zone d'étude......39

Tableau 8: Indice de faisabilité des modèles communautaires selon les RC et les ASC .....40

Tableau 9: Indice synthétique du niveau de connaissance en PF .........................41

Tableau 10 : Indice synthétique du niveau de connaissance en IST/VIH/SIDA ................42

Tableau 11: Indice synthétique du niveau de connaissance en maternité à moindre risque.43

Tableau 12: Indice synthétique du niveau de connaissance en Survie de l'enfant.............44

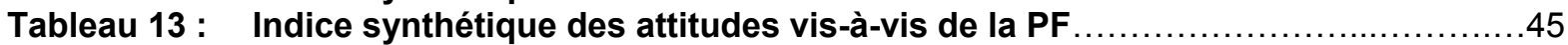

Tableau 14: Indice synthétique des attitudes vis-à-vis du VIH/SIDA $\ldots \ldots \ldots \ldots \ldots \ldots \ldots \ldots \ldots . \ldots 6$

Tableau 15 : Femmes qui ne sont pas sous méthode et qui n'ont pas l'intention d'en utiliser

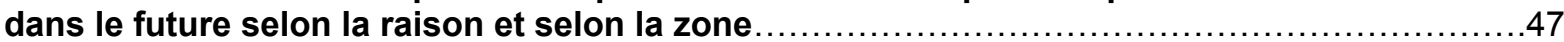

\section{Graphiques}

Graphique 1: $\quad$ Evolution du nombre d'activités des ASBC

Graphique 2: $\quad$ Pourcentage d'ASBC qui ne disposaient pas de produits PF et de médicaments au 19 moment de l'enquête

Graphique 3 : $\quad$ Répartition des ASBC selon leur opinion sur la formation reçue 20

Graphique 4 : $\quad$ Pourcentage de population-cible couverte selon le modèle et le sexe 24

Graphique 5: $\quad$ Pourcentage de femmes mariées qui ont utilisé une méthode contraceptive au cours des 25 12 derniers mois

Graphique 6: $\quad$ Pourcentage de femmes mariées qui utilisaient une méthode contraceptive au moment de 26 l'enquête

Graphique 7: $\quad$ Pourcentage d'hommes qui ont utilisé le préservatif au cours des 12 derniers mois 26

Graphique 8a: Pourcentage d'hommes mariés et non mariés qui ont déclaré avoir utilisé le préservatif au 27 cours des 12 derniers mois (post-intervention)

Graphique $8 \mathrm{~b}$ : $\quad$ Pourcentage d'hommes mariés et non mariés qui ont déclaré avoir utilisé le préservatif au 28 cours des 12 derniers mois (pré-intervention) 


\section{LISTE DES ABBREVIATIONS}

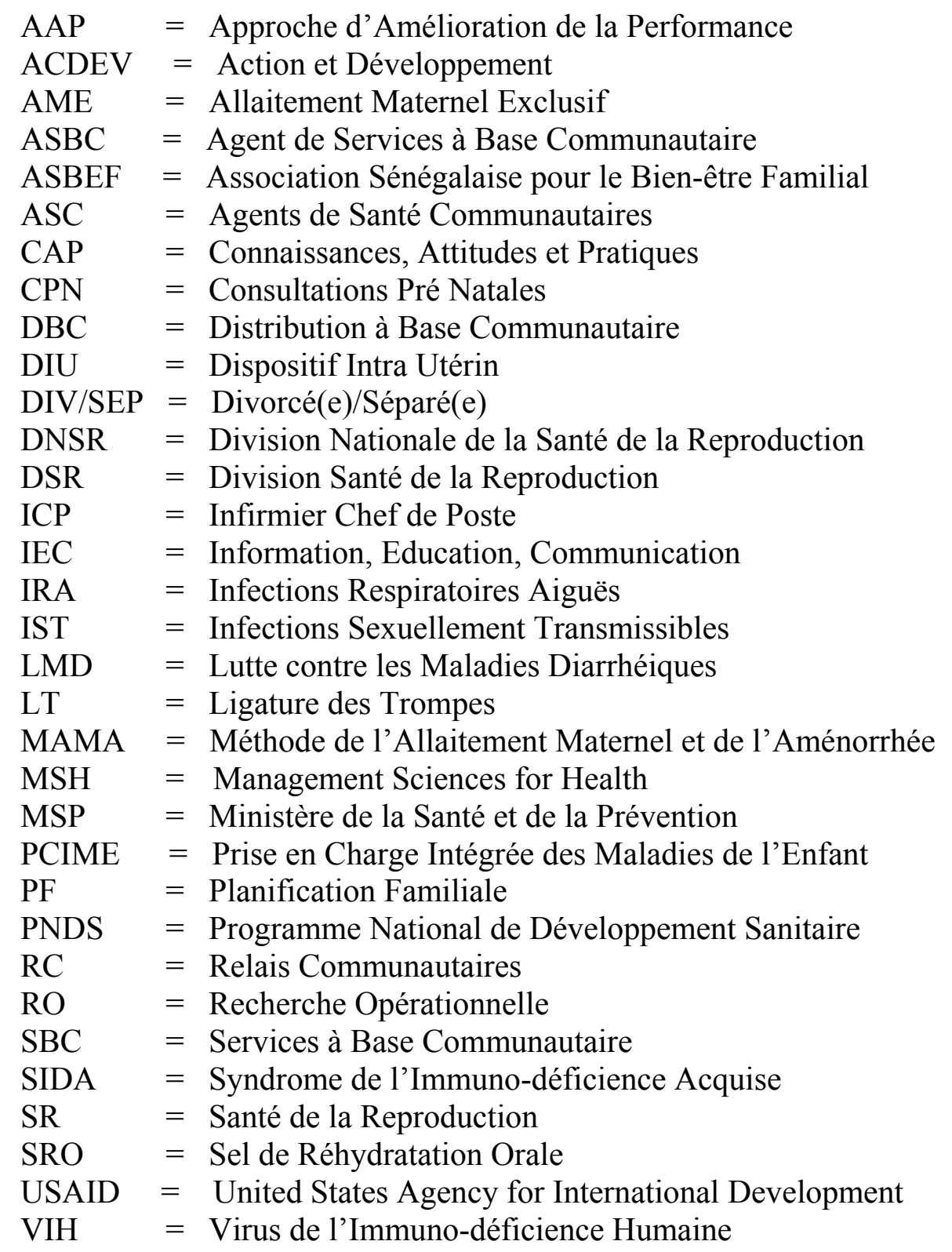




\section{REMERCIEMENTS}

Les auteurs remercient toutes les institutions et personnes qui ont permis la réalisation de cette étude.

En premier lieu, les responsables de la Division de la Santé de la Reproduction (DSR) et du Population Council dont l'intérêt et l'appui soutenus ont facilité l'exécution correcte de cette activité.

Sont également remerciés, pour leur contribution et/ou disponibilité, le Médecin-Chef du district sanitaire de Kébémer et toute l'équipe-cadre, les Infirmiers-Chefs de Poste, les autorités administratives locales, les populations du district ainsi que les partenaires que sont:

- Le Projet Santé Maternelle et Planification Familiale / Management Sciences for Health (MSH);

- Intrah-Prime II ;

- BASICS/Sénégal.

Nos remerciements s'adressent aussi :

- Au personnel d'appui de la DSR ;

- Au personnel d'appui du Population Council Dakar; et

- Au personnel de collecte et de saisie des données.

Finalement les auteurs expriment leur vive gratitude à l'USAID dont le financement et le soutien ont rendu possible la réalisation de cette étude. 


\section{INTRODUCTION}

Le présent rapport rend compte des résultats d'un projet des services à base communautaire (SBC). Le projet a été expérimenté pendant une période de 24 mois dans le district sanitaire de Kébémer. L'expérimentation proposée a été conçue pour tester et comparer trois manières d'offrir des services de santé de la reproduction ${ }^{1}$ aux communautés rurales du Sénégal du point de vue de leur degré d'acceptabilité, de faisabilité et d'efficacité.

Le projet comporte deux volets : un volet «Intervention» placé sous la responsabilité technique de Management Sciences for Health (MSH) portant sur l'exécution des activités des trois modèles communautaires ; et un volet «Recherche» confié au Population Council pour la réalisation des études de recherches opérationnelles afin de documenter le processus de mise en œuvre des interventions communautaires et mesurer le degré de faisabilité, d'acceptabilité et d'efficacité des modèles expérimentés. Le projet a été exécuté par la Division de la Santé de la Reproduction (DSR) du Ministère de la Santé et de la Prévention. Pour la mise en œuvre des activités des deux volets, il a bénéficié de l'assistance financière de l'USAID. D'autres partenaires tels que Intrah Prime II et Basics ont aussi contribué à l'intervention.

Le projet a été exécuté dans le district sanitaire de Kébémer situé à environ 160 kilomètres au nord de Dakar, dans la région de Louga. Le district sanitaire de Kébémer a une population d'environ 112000 habitants composés pour l'essentiel de Wolofs. La principale activité économique est l'agriculture. Ce district sanitaire est composé d'une commune qui abrite le Centre de santé, de 11 communautés rurales et d'environ 600 villages. Chaque communauté rurale abrite un Poste de santé, soit 11 Postes de santé pour le district.

\section{EXPOSE DU PROBLEME}

Les données de l'Enquête Démographique et de Santé (EDS III, 1997)² montrent l'existence au Sénégal d'un grand nombre d'utilisateurs potentiels d'informations et de services de PF et de santé de la reproduction qui ne sont pas servis. Le fait que ces groupes ne soient pas correctement couverts par le programme actuel basé dans les points de prestation de services (PPS) cliniques, laisse penser qu'il y a un véritable problème d'accès aux informations et aux services de base disponibles en matière de santé de la reproduction. En effet, l'Analyse Situationnelle menée en $1998^{3}$ dans les 10 régions du Sénégal avait révélé de nombreux problèmes ayant trait à la qualité des soins dispensés dans les PPS de santé de la reproduction. En particulier, le temps d'attente était jugé trop long par les clientes, surtout au vu de la brève interaction avec les prestataires de services; et la plupart des clientes ne recevaient pas toutes les informations dont elles avaient besoin. Les clientes qui fréquentaient les PPS pour des visites prénatales ne recevaient que peu de conseils ou d'informations. Enfin, l'étude avait révélé que même si les prestataires de services avaient de solides connaissances en matière de IST/VIH/SIDA, ils ne discutaient / recommandaient pas de préservatifs aux clientes.

\footnotetext{
${ }^{1}$ Les quatre composantes de la Santé de Reproduction retenues dans le cadre de ce projet sont : (1) La PF, (2) les IST/SIDA, (3) la Maternité à Moindre Risque, et (4) la Survie de l'Enfant.

${ }^{2}$ Direction de la Prévision et de la Statistique et Macro International Inc., 1997 : Enquête Démographique et de Santé au Sénégal (EDS-III), 238 pages

${ }^{3}$ Ministère de la Santé et de l'Action Sociale et Population Council, 2000 ( $2^{\text {nde }}$ édition) : Évolution des Services de Santé de la Reproduction et Planification Familiale de 1994 à 1998, 60 pages.
} 
Dans ce contexte, le MSP a essayé d'identifier d'autres stratégies pour l'offre de services et d'informations de qualité sur la santé de la reproduction en milieu rural comme en milieu urbain. Cet intérêt croissant du gouvernement du Sénégal pour l'élaboration et l'expérimentation d'autres modèles de prestation de services communautaires s'est traduit d'une part, par le financement de plusieurs voyages d'étude de personnes-ressources du MSP au Mali, au Kenya et en Indonésie pour en savoir davantage sur les expériences de ces pays en matière de Distribution à Base Communautaire (DBC) ; et d'autre part, par l'organisation en juin 1999 à Dakar, d'un atelier national d'orientation de trois jours sur la stratégie d'offre de services à base communautaire pour présenter et discuter les résultats et enseignements tirés d'expériences antérieures en Afrique sub-saharienne, mais aussi pour définir les bases d'un modèle standard de services à base communautaire (SBC) au Sénégal. Les recommandations de cet atelier ont surtout mis l'accent sur la nécessité de définir un modèle standard d'agents SBC en tenant compte du contexte socioculturel du Sénégal. Par la suite, l'idée de comparer les deux approches SBC expérimentées a été adoptée en juin $2000^{4}$ par le comité technique de suivi de l'application des recommandations dudit atelier.

En effet, l'utilité et la nécessité des programmes SBC pour le Sénégal ne sont plus à démontrer tant l'approche SBC est compatible avec la politique de décentralisation des services de santé entreprise par le gouvernement. Mais la multiplicité des initiatives et actions concrètes $^{5}$ non coordonnées concourent à dire que le moment est venu, pour le secteur public, de créer et de développer un modèle de SBC qui soit un cadre de référence adapté au contexte sénégalais. Il permettrait en même temps de mieux canaliser les actions des différents partenaires au développement et assurer une plus grande disponibilité et accessibilité des services de santé de la reproduction. En effet, les expériences vécues dans plusieurs pays africains ont montré que les programmes ${ }^{6}$ de DBC peuvent améliorer l'accessibilité, la qualité et l'acceptabilité des services de santé de la reproduction. Mais ils peuvent également échouer à cause de leur incapacité à s'adapter à l'environnement local ou à susciter l'engagement des communautés concernées. Les enseignements tirés de l'expérimentation font l'objet de ce rapport. Ils devraient permettre au MSP d'apporter des réponses aux questions d'adaptation des composantes majeures de l'approche SBC et de faire une option par rapport à chacun de ces deux modèles de prestation de services SR avant de s'engager dans la mise en œuvre d'un programme SBC d'envergure nationale.

\footnotetext{
${ }^{4}$ Les représentants des organisations suivantes ont participé à cette réunion technique : USAID/Sénégal, MSH (chargé de la mise en œuvre du projet bilatéral de l'USAID), le SNSR, l'ASBEF, ADEMAS et Population Council

5 Plusieurs initiatives et activités SBC sont en cours d'exécution avec l'UNFPA à Tambacounda et Kédougou, la GTZ à Kolda, Africaire à Kaolack, l'ASBEF dans plusieurs zones urbaines, et SANFAM au niveau des entreprises et groupements féminins. En outre, ADEMAS, un programme de marketing social, couvre les zones urbaines et semi-urbaines du pays; et plusieurs bailleurs de fonds dont l'USAID, le FNUAP et la Banque Mondiale sont également engagés à financer les activités de SBC dans les court et moyen termes.

${ }^{6}$ Phillips, J. \& W. Green, 1993. « Distribution Communautaire des Services de Planification Familiale en Afrique : Leçons tirées des activités de Recherche Opérationnelle » The population Council, New York, USA
} 


\section{METHODOLOGIE}

\section{Objectif général}

L'objectif général de ce projet de recherches opérationnelles est de contribuer à l'élaboration de stratégies alternatives pour l'amélioration de la disponibilité et de l'accessibilité des informations et des services de qualité en matière de santé de la reproduction (SR) au Sénégal.

\section{Objectifs spécifiques}

1. Tester trois modèles de prestations de services à base communautaire en matière de santé de la reproduction;

2. Comparer les trois modèles de services à base communautaire expérimentés du point de vue de leur acceptabilité, de leur faisabilité, de leur efficacité, de leur performance et de leur coût et rentabilité ;

3. Faire des recommandations par rapport au développement d'un modèle de référence de SBC pour le Sénégal.

\section{Questions de recherche}

- La redynamisation des cases de santé est-elle suffisante pour accroître la demande et l'utilisation des services de SR et répondre aux besoins de SR des clientes issues des villages satellites de la case de santé et des villages de la case de santé?

- L'introduction des relais communautaires est-elle plus efficace que la redynamisation des cases de santé dans l'augmentation des connaissances et l'utilisation des services de SR ?

- Quels sont les coûts et la rentabilité relatifs de chaque modèle communautaire ?

\section{Hypothèses}

Les différentes hypothèses à vérifier, après l'exécution des interventions décrites dans la section suivante, sont :

1. Les niveaux des connaissances et d'utilisation des services de PF et des autres services de SR après les interventions seront meilleurs à ceux qu'ils l'étaient avant ces interventions.

2. Les femmes vivant dans les villages où les interventions communautaires ont été introduites connaîtront mieux et utiliseront davantage les services de santé de la reproduction que celles vivant dans les villages couverts par l'intervention qui porte sur le renforcement des formations sanitaires.

3. Les femmes vivant dans les villages où les relais communautaires font des visites à domicile connaîtront mieux et utiliseront davantage les services de santé de la reproduction que celles vivant dans les villages où les agents de santé communautaires restent au niveau des cases de santé.

4. Les performances des relais communautaires qui font des visites à domicile seront meilleures que celles des agents de santé communautaires des cases de santé.

5. Le modèle dans lequel les relais communautaires font des visites à domicile sera plus rentable que les autres modèles expérimentés.

Ces hypothèses seront vérifiées à l'aide de données empiriques collectées à partir des études de RO menées pendant la période du projet. 


\section{Description des interventions}

Le premier modèle communautaire repose sur l'utilisation des Agents de Santé Communautaires qui travaillent dans les Cases de santé en plus du renforcement de la capacité de fonctionnement des Postes de santé. Le second modèle porte sur l'introduction de Relais Communautaires (RC) dans les aires de responsabilité des Cases de santé non fonctionnelles en plus du renforcement de la capacité de fonctionnement des Postes de santé. Enfin, le troisième modèle, le modèle de comparaison, consiste à renforcer la capacité de fonctionnement du Centre et des Postes de santé seulement.

- Intervention d'offre de services de SR basée sur le renforcement de la capacité technique des Cases de santé fonctionnelles

Ce modèle d'intervention repose sur l'utilisation des agents de santé communautaires des Cases de Santé fonctionnelles pour offrir des informations et services de SR après qu'ils aient reçu une formation appropriée pour renforcer leur capacité technique, et que les cases de santé aient été doté du minimum d'équipements et de produits nécessaires pour la fourniture de ces services de SR.

Les Cases de Santé sont des structures créées et gérées par la communauté. Elles se situent en dessous des postes de santé, le niveau le plus bas de la pyramide sanitaire. Elles ont un rôle essentiellement préventif (PNDS, 1997) ${ }^{7}$. Chaque Case de Santé dispose d'un personnel composé d'au moins trois Agents de Santé Communautaires (ASC): matrone, hygiéniste, relais IEC ou promotrice. Ces ASC sont des membres de la communauté qui travaillent à mitemps sous la direction et avec l'appui des comités de santé. Ils sont choisis par la communauté. L'Infirmier Chef de Poste (ICP) de chaque Poste de Santé est chargé de la supervision des Cases de Santé qui se trouvent dans sa zone d'action. Les ASC sont formés pour offrir des soins de santé primaires de base et des soins préventifs. Dans le district de Kébémer, ils fournissent des services tels que le traitement de la diarrhée par réhydratation orale, l'éducation nutritionnelle, les accouchements, le traitement du paludisme et la prévention des maladies transmissibles, le counseling et les visites à domicile, ainsi que la gestion des médicaments au niveau des cases de santé. Ils ont reçu des rudiments de formation en planification familiale et en régulation des naissances, mais ne sont pas habilités à fournir ces services pour lesquels ils se limitent jusqu'ici à donner les informations et faire des références.

Les Cases de santé existent depuis un certain nombre d'années, et certaines d'entre elles sont actuellement fonctionnelles alors que d'autres ne le sont pas. Pour être considérée comme fonctionnelle par le SNSR, une Case de Santé doit nécessairement avoir : (1) un personnel qualifié et actif sur place, (2) des infrastructures physiques, (3) des équipements et des produits, et (4) un comité de santé.

L'offre de services de SR à partir des Cases de Santé n'a été mise en œuvre que dans les Cases de Santé actuellement fonctionnelles. Elle consiste à donner au personnel de ces Cases de Santé fonctionnelles une nouvelle formation axée sur des informations et services de santé de la reproduction, et à s'assurer que le minimum d'équipements et de produits nécessaires pour l'offre de ces services est disponible. Elle traduit une volonté du gouvernement d'offrir

\footnotetext{
${ }^{7}$ Ministère de la Santé Publique et de l'Action Sociale, Juin 1997: Plan National de Développement Sanitaire et Social du Sénégal (1998-2007), 54 pages
} 
des services de santé de la reproduction au niveau le plus périphérique du système de santé, c'est-à-dire au niveau des Cases de Santé pourvues en personnel formé et en équipements. Ce modèle repose sur l'hypothèse que même si les Cases de Santé fonctionnelles ont prouvé leur aptitude à offrir des services curatifs, d'autres inputs, en l'occurrence la mise à niveau du personnel, le recyclage et l'approvisionnement en produits, sont nécessaires pour leur permettre d'offrir, de manière adéquate, des services de santé de la reproduction, car ceux-ci sont plus sensibles d'un point de vue culturel et demandent par conséquent des compétences dans divers domaines. Dans le cadre de cette intervention, les activités suivantes ont été menées :

La formation du personnel des Cases de Santé: Quinze (15) Cases de santé fonctionnelles ont été retenues pour l'expérimentation de ce modèle. Au niveau de chaque Case de santé, deux ASC ont été choisis comme personnel du projet SBC. Ces ASC ont reçu une formation de 12 jours basée sur un module de formation portant sur les quatre composantes de la SR retenues dans le cadre du projet. Un accent particulier a été également mis sur les techniques d'IEC (causerie éducative, visite à domicile, entretien/counseling, utilisation des supports), la référence et le suivi des clients, et sur la gestion des services à base communautaires (gestion des ressources matérielles et des produits, gestion financière et gestion des statistiques de services). Une session de recyclage de 5 jours a été organisée six mois après le démarrage des activités des ASC.

L'équipement des Cases de Santé : L'équipement des Cases de santé a été fait en tenant compte des besoins spécifiques de chacune d'entre elles. Pour l'ensemble des 15 Cases de santé, il a porté sur la fourniture de 10 armoires, 15 tables, 10 tables d'accouchement, 5 boîtes d'accouchement, 10 plateaux inox, 15 paires de gants, 15 haricots inox MM, 15 brûleurs de gaz, 30 bassines de 20 litres, 15 chaises en plastiques, 15 lampes-tempête et 15 supports de gaz.

La dotation en produits des Cases de Santé : Elle a été faite en deux temps. La première dotation a été faite par le district sanitaire de Kébémer. Dans ce cadre, chaque ICP a reçu 60 cycles de pilules (dont 45 cycles de Loféménal et 15 cycles d'Ovrette), 300 comprimés de spermicide, 500 unités de préservatifs et 100 sachets de Sel de Réhydratation Orale (SRO) à partager aux Cases de santé qu'il supervise. La seconde dotation a été assurée par l'état. Chaque Case de santé a reçu 1 boîte de 1000 comprimés de chloroquine, 1 boîte de 1000 comprimés de paracétamol, 1 boîte de 600 comprimés de Mébendazole, 1 boîte de 1000 comprimés de fer/folate, 1 boîte de 500 capsules de Vitamine A 200000 UI et 2 boîtes de 50 sachets de SRO.

- Intervention d'offre de services de SR basée sur l'introduction des Relais Communautaires dans les aires des Cases de santé non fonctionnelles

Dans ce modèle, deux volontaires membres de la communauté et appelés Relais Communautaires (RC) sont recrutés pour travailler dans la zone d'action d'une Case de Santé non fonctionnelle. Considérant que l'utilisation des services de santé de la reproduction est une question culturellement plus sensible que celle des soins de santé primaires, et que la vulgarisation par le porte-à-porte a prouvé son efficacité dans les zones où ces services ne sont pas largement utilisés par les populations, ce modèle repose sur l'hypothèse selon laquelle un système communautaire basé sur une approche de vulgarisation pro-active peut être plus efficace qu'un système communautaire basé sur des structures de santé fixes. 
Un total de $30 \mathrm{RC}$ a été choisi par la communauté comme agents de distribution du projet $\mathrm{SBC}$ en raison de deux RC par zone couverte ou supposée d'action d'une Case de santé non fonctionnelle. Ce choix a été fait sur la base des critères de référence suivants définis au préalable par les leaders et les membres de la communauté : genre (homme ou femme), âge (20 à 45 ans), résidence (être résident dans la communauté), niveau d'instruction (être instruit ou alphabétisé), statut matrimonial (être marié de préférence), et autres (être disponible, être dynamique, être choisi par la communauté, être crédible, sociable, discret, tolérant et être volontaire). La zone d'expérimentation de ce modèle communautaire compte trois Postes de santé dirigés chacun par un ICP. A chaque ICP est rattaché 5 binômes de RC qu'il supervise techniquement. A la différence des ASC qui sont fixes au niveau des Cases de santé, les RC sont plutôt mobiles.

Pour faciliter leurs déplacements, chaque binôme de $\mathrm{RC}$ a reçu du projet un cheval et une charrette dont ils assurent l'entretien. Les RC ont reçu la même formation de base, la même session de recyclage et les mêmes dotations de produits $\mathrm{SBC}$ selon les mêmes procédures que les ASC.

\section{- Intervention de renforcement du Centre et des Postes de Santé}

Un préalable pour tester les interventions communautaires consiste à s'assurer que les PPS cliniques (ici, le Centre et les Postes de Santé du district) sont en mesure de répondre à une augmentation possible de la demande de services en santé de la reproduction. Dans ce cadre, une rapide évaluation des besoins en formation du personnel et en équipements et produits, a été effectuée dans les 10 Postes de Santé et le Centre de Santé de Kébémer. Suite à cette évaluation des besoins, les actions concrètes suivantes ont été réalisées au titre de ce renforcement des Postes et du Centre de Santé :

* la formation du personnel de santé : une session d'orientation de 12 jours de l'équipe cadre du district et des $10 \mathrm{ICP}$ du district sanitaire sur les quatre composantes de la SR prises en compte par le projet a été organisée.

* l'amélioration du système de supervision par la formation de l'équipe-cadre du district et des 10 ICP du district sanitaire sur l'approche de l'amélioration des performances (AAP) et la dotation en grilles et plans de supervision comme outils de supervision.

* la dotation des 6 postes de santé des zones expérimentales de fiches de synthèse des activités SBC. 
Tableau 1 : Types de services disponibles selon le niveau de prestation et la composante de SR

\begin{tabular}{|c|c|c|c|}
\hline $\begin{array}{l}\text { Composante de } \\
\text { la SR }\end{array}$ & $\begin{array}{l}\text { Centre de santé } \\
\text { Poste de santé }\end{array}$ & Case de santé & Relais communautaire \\
\hline PF & $\begin{array}{l}\text { Counseling sur les } \\
\text { méthodes } \\
\text { contraceptives, offre de } \\
\text { méthode contraceptive, } \\
\text { réapprovisionnement et } \\
\text { traitement des effets } \\
\text { secondaires }\end{array}$ & $\begin{array}{l}\text { Informations sur les méthodes } \\
\text { contraceptives, } \\
\text { réapprovisionnement en pilules, } \\
\text { vente de spermicide et } \\
\text { préservatifs, références pour la } \\
\text { PF }\end{array}$ & $\begin{array}{l}\text { Informations sur les méthodes } \\
\text { contraceptives, réapprovisionnement en } \\
\text { pilules, vente de spermicide et } \\
\text { préservatifs, références pour la PF }\end{array}$ \\
\hline IST/VIH/SIDA & $\begin{array}{l}\text { Informations sur les } \\
\text { IST/SIDA et les moyens } \\
\text { de protection, dépistage } \\
\text { et traitement des } \\
\text { infections. }\end{array}$ & $\begin{array}{l}\text { Informations sur les symptômes } \\
\text { d'IST/SIDA et les moyens de } \\
\text { prévention, références }\end{array}$ & $\begin{array}{l}\text { Informations sur les symptômes } \\
\text { d'IST/SIDA et les moyens de prévention, } \\
\text { références }\end{array}$ \\
\hline $\begin{array}{l}\text { Maternité à } \\
\text { moindre risque }\end{array}$ & $\begin{array}{l}\text { Consultation des } \\
\text { femmes enceintes et } \\
\text { suivi médical de la } \\
\text { grossesse, assister les } \\
\text { accouchements, suivi } \\
\text { postnatal }\end{array}$ & $\begin{array}{l}\text { Sensibilisation et informations } \\
\text { sur les CPN, référence et suivi } \\
\text { des femmes enceintes, vente de } \\
\text { Fer aux femmes enceintes }\end{array}$ & $\begin{array}{l}\text { Sensibilisation et informations sur les } \\
\text { CPN, référence et suivi des femmes } \\
\text { enceintes, vente de Fer aux femmes } \\
\text { enceintes }\end{array}$ \\
\hline $\begin{array}{l}\text { Survie de } \\
\text { l'enfant }\end{array}$ & $\begin{array}{l}\text { Prise en charge intégrée } \\
\text { des maladies de l'enfant } \\
(\text { PCIME) }\end{array}$ & $\begin{array}{l}\text { Sensibilisation et information sur } \\
\text { les pratiques néfastes à la survie } \\
\text { de l'enfant; référence des enfants } \\
\text { pour la vaccination et la pesée; } \\
\text { causeries sur le paludisme et } \\
\text { vente de chloroquine ; causeries } \\
\text { sur la diarrhée et vente de sachets } \\
\text { de SRO ; vente de gélules de Vit. } \\
\text { A ; références. }\end{array}$ & $\begin{array}{l}\text { Sensibilisation et information sur les } \\
\text { pratiques néfastes à la survie de l'enfant ; } \\
\text { référence des enfants pour la vaccination } \\
\text { et la pesée ; causeries sur le paludisme et } \\
\text { vente de chloroquine ; causeries sur la } \\
\text { diarrhée et vente de sachets de SRO; } \\
\text { vente de gélules de Vit. A ; références. }\end{array}$ \\
\hline
\end{tabular}

Les trois modèles communautaires d'offre de services de SR expérimentés dans le cadre de cette étude sont une combinaison de ces trois types d'intervention:

* le renforcement de la capacité des Cases de santé fonctionnelles en plus du renforcement de la capacité de fonctionnement des postes de santé;

* l'introduction des Relais Communautaires dans des aires de responsabilité de Cases de santé non fonctionnelles en plus du renforcement de la capacité de fonctionnement des postes de santé ;

* le renforcement du centre et des postes de santé seulement.

Le tableau 1 résume les services de santé de la reproduction qui ont été mis à la disposition des clients à travers les trois modèles d'offre de services.

\section{Processus de mise en ouvre des modèles communautaires}

Il se résume à travers l'exécution des principales activités suivantes :

- Les concertations au niveau national et au niveau District : La concertation au niveau national a regroupé toutes les parties ${ }^{8}$ prenantes du projet SBC et d'autres agences de collaboration intéressées par l'expérimentation envisagée. Le but de cette première réunion était de présenter le projet $\mathrm{SBC}$ et de constituer un comité technique de suivi des activités du projet. Une seconde concertation sur le projet SBC a eu lieu au niveau

\footnotetext{
${ }^{8}$ Le MSP, le district sanitaire de Kébémer, l’USAID, MSH et Population Council
} 
District. Elle a porté sur la définition des modalités pratiques de mise en œuvre du projet et le découpage opérationnel du district pour les besoins de la recherche.

- Les concertations au niveau communautaire : Des rencontres ont été organisées avec les communautés concernées par le projet SBC avant son démarrage.

Au niveau de chaque réunion, le projet fut présenté à un auditoire composé des autorités administratives, des présidents de communautés rurales, des chefs de villages et des représentants des groupements de femmes, de jeunes et d'émigrés. Lors de ces concertations, les représentants des communautés ont défini les critères de sélection des relais communautaires. Au niveau des sites expérimentaux avec $\mathrm{RC}$, des réunions ont été tenues pour préciser davantage les modalités pratiques de mise en œuvre de l'intervention et apprécier la sélection des RC.

- L'atelier d'élaboration du curriculum de formation et d'orientation des formateurs : Sur la base de documents nationaux et de curricula de formation d'autres pays ${ }^{9}$ ayant expérimenté la DBC, une équipe composée de représentants du MSP, de la Région Médicale de Louga, de l'équipe-cadre du district, des parties prenantes de l'intervention et d'autres institutions intervenant dans la santé de la reproduction ${ }^{10}$, a élaboré un curriculum de formation des agents de services à base communautaire de santé de la reproduction. Cet atelier a été organisé pour deux semaines au niveau du district sanitaire. En prenant part à l'élaboration du curriculum de formation des ASBC, les formateurs du MSP ont été, en même temps, imprégnés de son contenu. Ce curriculum comporte 4 modules et 10 sous modules correspondant à 30 sessions.

* Module I :La stratégie d'offre de Services à Base Communautaire (SBC) dans le cadre de la politique de SR au Sénégal.

* Module II: Les composantes de l'offre de services à base communautaire de SR.

- Sous module1 : L'anatomie et la physiologie de la reproduction ;

- Sous module 2: La contraception;

- Sous module 3: Les Infections Sexuellement Transmissibles et le VIH/SIDA ;

- Sous module 4 : L'infécondité/infertilité ;

- Sous module 5: La survie de l'enfant et la promotion des pratiques clés dans la communauté ;

○ Sous module 6: La maternité à moindre risque ;

- Sous module 7 : Les pratiques néfastes;

○ Sous module 8: L'hygiène/assainissement ;

- Sous module 9: Le paludisme ;

- Sous module 10: La référence et le suivi ;

* Module III: Information, Education, Communication (IEC).

* Module IV : Gestion des services à base communautaire de SR.

- L'atelier de formation des superviseurs : Suite à l'élaboration du curriculum et à l'orientation des formateurs du MSP, ces derniers ont procédé à la formation des 10 Infirmiers-Chefs de Poste (ICP) sur le contenu de ce curriculum. Dans cette activité de formation, les formateurs du MSP ont bénéficié de l'appui de la Région Médicale de

\footnotetext{
${ }^{9}$ Mali, Cameroun.

${ }^{10}$ Intrah/Prime, ASBEF, Sanfam, Ademas, GTZ.
} 
Louga et d'autres institutions intervenant dans le domaine de la SR. De manière générale, l'objectif de cet atelier était de préparer les ICP à assurer la formation et la supervision des ASBC. La formation des 10 ICP a duré 12 jours.

- Les réunions de planification et de suivi des activités préparatoires : Les réunions tenues au niveau central ont constitué des occasions pour les parties prenantes du projet d'évaluer les étapes franchies, de réfléchir sur les prochaines activités à réaliser, de définir les modalités pratiques de mise en œuvre, de situer les responsabilités et de fixer des délais d'exécution. Les principales décisions relatives à l'intervention ont été prises au cours de ces réunions. Sous la présidence du MSP, ces réunions ont noté la présence des représentants de toutes les parties prenantes de l'intervention. Au niveau district des réunions de planification des activités ont été également tenues pour étudier la mise en œuvre, au niveau local, des décisions prises lors des réunions de coordination au niveau central. Elles ont regroupé, outre l'équipe-cadre du district, le personnel médical du district, les parties prenantes de l'intervention, les autorités administratives du district sanitaire, les leaders communautaires, les présidents de comités de santé, les représentants de groupements de femmes, de jeunes et d'émigrés et les représentants des agences de développement intervenant au niveau du district.

Outre ces deux instances, des réunions ont été également organisées par le comité technique pour réfléchir, dans un cadre plus restreint, des activités de préparation de l'intervention. Ces réunions étaient tenues tantôt au niveau central tantôt au niveau du district.

Les réunions de coordination au niveau central comme les réunions de planification des activités SBC au niveau district ne sont pas des instances convoquées expressément pour les activités SBC. Ce sont des activités inscrites dans les fonctionnements respectifs du MSP et du district. Lors de ces réunions, les activités SBC étaient inscrites à l'ordre du jour au même titre que d'autres activités que coordonnait le MSP ou le district. Quant aux réunions du comité technique, elles portaient exclusivement sur les activités du projet SBC.

- L'atelier de formation des ASBC: La formation des agents SBC a eu lieu au niveau district et s'est étalée sur une période de deux semaines. Trois sites ont été retenus en fonction du nombre et du lieu de résidence des ASBC. La formation a été faite simultanément au niveau des trois sites. Elle a été animée par les 10 Infirmiers-Chefs de Postes du district, appuyés par l'équipe-cadre du district et les représentants de la Région Médicale de Louga et du MSP qui avaient participé à l'élaboration du curriculum de formation des ASBC. Au total $30 \mathrm{RC}$ et 30 ASC ont été formés suivant les mêmes modules du curriculum de formation.

- L'orientation des ASBC sur le remplissage des outils de gestion : En principe, l'orientation des ASBC sur le remplissage des outils de collecte des statistiques de services devait être faite au moment de la formation des ASBC. Mais des contraintes logistiques (non-disponibilité de la version finale des outils de collecte) ont fait que cette activité a été différée. Dans sa mise en œuvre, un atelier d'orientation a été organisé au niveau de chaque site expérimental ( 3 sites ASC et 3 sites RC) et chaque ASBC a reçu un jeu des outils de gestion. En présence de l'ICP de chaque site et du représentant du MSP, l'orientation des ASBC a été faite par le Population Council chargé du volet recherche. L'objectif visé par cette orientation était de permettre aux ASBC de tenir correctement 
les statistiques de leurs activités respectives dans le cadre du projet SBC. Un jour-atelier a été retenu pour l'orientation des ASBC. Dans la pratique ce temps imparti s'est avéré très insuffisant pour permettre aux ASBC de bien assimiler les enseignements reçus.

- La mise à disposition des équipements et matériels de travail : Conformément aux objectifs de l'étude et à la nature des modèles communautaires à expérimenter, le projet s'est investi dans l'achat d'équipements pour les Cases de santé. Comme indiqué dans les sections qui précèdent, la liste des équipements et matériels donnés au niveau des cases de santé inclut des armoires, tables, tables d'accouchement, boîtes d'accouchement, plateaux inox, paires de gants, haricots inox MM, brûleurs de gaz, bassines de 20 litres, chaises en plastiques, lampes-tempête et supports de gaz au niveau des Cases de santé. Les résultats de l'Analyse Situationnelle à minima ont servi de base aux autorités médicales du district pour déterminer les besoins prioritaires selon les Cases de santé et les quantités de matériels nécessaires pour assurer leur fonctionnalité. Au niveau des RC cette logistique comprend les charrettes et chevaux, et les trousses de travail des ASBC contenant tous les échantillons des méthodes contraceptives modernes (spermicide, préservatif, pilules, injectables, DIU, Norplant), et les matériels IEC (mannequin, cartes IST, dépliants).

Comme dans toute intervention du genre, la mise en œuvre des activités du projet ne s'est pas fait sans problèmes. Des facteurs de différentes natures et d'origines diverses ont certainement amoindri l'effet des interventions. Ces facteurs sont en général des déviations ou biais par rapport au schéma d'expérimentation défini dans le protocole technique ; mais aussi des facteurs liés aux difficultés d'exécution de certaines activités par rapport aux conditions de terrain. Ils se résument:

* à la faiblesse ou absence de moyens de déplacement pour les RC : cette situation est née du mauvais état de santé des chevaux en général qui a abouti au décès de trois chevaux;

* au non-respect du rythme de supervision tel que décrit dans le protocole technique : au cours de l'expérimentation, la supervision a été faite au gré des ICP au lieu d'une supervision mensuelle pendant les 6 premiers mois du projet, et une supervision trimestrielle au-delà de cette période.

* au non-respect de la périodicité des sessions de recyclage : dans la pratique, une seule session de recyclage contre trois a été organisée pendant les 12 mois d'expérimentation ;

* aux besoins en équipements non satisfaits au niveau du Centre et des Postes de santé du fait de contraintes budgétaires; mais ce manquement ne semble pas avoir affecté les demandes de services au niveau des formations sanitaires ;

* à l'absence d'un point focal chargé de la coordination quotidienne des activités SBC sur le terrain qui aurait pu palier aux insuffisances liées au faible niveau de supervision ;

* à la faible contribution des comités de santé dans la mise en œuvre des activités du projet ;

* au non-respect des critères de sélection dans le choix des RC dans certaines zones du projet: environ 30\% des RC et $44 \%$ des ASC n'avaient aucun niveau d'instruction et la plupart des ASC étaient trop âgés pour être performants. 


\section{Procédé de recherche}

Le procédé de recherche représente le plan du chercheur pour minimiser les sources d'erreurs, répondre aux questions de recherche et vérifier les hypothèses.

Pour la mise en œuvre du projet, le district sanitaire de Kébémer a été divisé en trois zones géographiques concentriques autour du Centre de santé. Au niveau de chaque zone, un modèle de services de SR a été expérimenté. La sélection d'une zone géographique pour l'expérimentation d'un modèle a été faite de façon raisonnée en tenant compte de critères de fonctionnalité des Cases de santé :

- La première zone géographique, la plus excentrée par rapport au Centre de santé, a été retenue pour l'expérimentation du modèle communautaire qui associe l'introduction des Relais Communautaires au renforcement de la capacité fonctionnelle des Postes de santé $(\mathrm{Xc}+\mathrm{X} 1)$. Cette zone compte trois Postes de Santé et correspond à la zone la plus inaccessible, caractérisée par la plus forte proportion de Cases de Santé non fonctionnelles dans le district sanitaire.

- La deuxième zone géographique contient également trois Postes de Santé. Cette zone se caractérise par l'existence d'une plus grande proportion de Cases de Santé fonctionnelles. Elle a été retenue pour l'expérimentation du modèle communautaire qui associe le renforcement de la capacité fonctionnelle des Cases des Santé à celui des Postes de Santé $(\mathrm{Xc}+\mathrm{X} 2)$.

- La troisième zone géographique regroupe quatre Postes de Santé et le Centre de Santé du district. La quasi-totalité des Cases de Santé contenues dans cette zone est fonctionnelle. Elle a été retenue comme zone de comparaison (Xc). Le schéma suivant décrit le procédé de recherche utilisé pour la mise en œuvre de l'expérimentation.

Zone expérimentale avec $\mathrm{RC}$

$\mathrm{Xc}+\mathrm{X} 1$

$\mathrm{O} 2$

Zone expérimentale avec ASC

Zone de comparaison
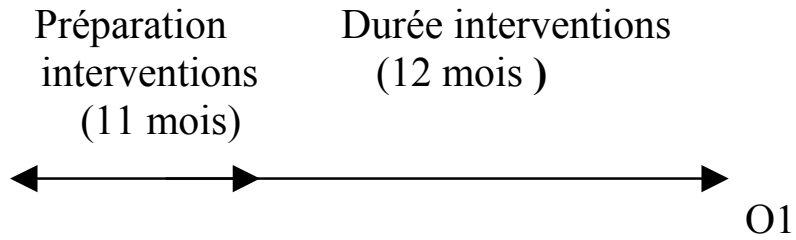

$\mathrm{O} 3$

$\mathrm{Xc}+\mathrm{X} 2 \quad \mathrm{O} 4$

O5

$\mathrm{Xc}$

O6

Sur ce schéma :

$\mathrm{O} 1, \mathrm{O} 3$ et $\mathrm{O} 5=$ Mesures de base des variables dépendantes, qui traduisent la situation des indicateurs en matière de SR avant l'exécution des modèles SBC ;

$\mathrm{O} 2$, $\mathrm{O} 4$ et $\mathrm{O} 6=$ Mesures des variables dépendantes qui traduisent la situation des indicateurs en matière de SR après l'exécution des modèles SBC;

$\mathrm{Xc}=$ Activités de renforcement des formations sanitaires : Centre et Postes de santé

$\mathrm{X} 1=$ Introduction des Relais Communautaires ;

$\mathrm{X} 2=$ Renforcement de la capacité fonctionnelle des Cases de santé.

L'efficacité de chaque modèle expérimenté est évaluée sur la base de l'évolution qu'il aura induite sur les niveaux de connaissances et d'utilisation des services de SR de la reproduction des populations desservies. Pour mesurer cette évolution, l'on a comparé les niveaux de ces 
indicateurs obtenus des données des enquêtes CAP avant l'intervention (O1, O3 et O5) et après l'intervention $(\mathrm{O} 2, \mathrm{O} 4$ et $\mathrm{O} 6)$. Toutes choses étant égales par ailleurs, les variations entre ces deux séries de mesures reflètent les changements imputables à chaque modèle particulier. Toute variation statistiquement significative $(\mathrm{O} 2-\mathrm{O} 1)$ ou $(\mathrm{O} 4-\mathrm{O} 3)$ est attribuée au modèle communautaire expérimenté si, concomitamment, (O6 - O5) n'atteste pas d'une variation statistiquement significative. Mais, si à une variation $(\mathrm{O} 2-\mathrm{O} 1)$ ou $(\mathrm{O} 4-$ O3) coïncide une variation (O6 - O5) similaire, on ne peut conclure sur un effet quelconque du modèle expérimenté. Dans ce cas de figure, il faudrait faire recours à des facteurs exogènes à l'intervention pour expliquer les variations constatées.

\section{Echantillonnage}

Trois unités d'enquêtes sont utilisées pour évaluer les objectifs du projet: les ASBC, les ICP superviseurs des ASBC et la population cible bénéficiaire des interventions. La méthode de sondage utilisée a été fonction du type d'unité statistique.

- Les ASBC : Dans le cadre des évaluations post-test, la quasi-totalité des RC et ASC (environ 87\%) ont été enquêtés. Sur l'ensemble des 30 RC et 30 ASC qui sont utilisés dans le cadre du projet, 52 d'entre eux, 25 ASC et 27 RC, ont pu être interviewés. Pour les 5 ASC non interviewés, les raisons suivantes expliquent cette situation: démission sur ordre du mari, migrations et voyages en dehors en dehors de la zone d'étude. Les 3 cas de RC non interviewés sont également liés à des raisons de migrations vers les centres urbains et de maladie. De manière générale, les ASBC sont mariés et ont pour occupation principale l'agriculture et les travaux domestiques. Une infime minorité d'entre eux s'adonne au petit commerce. La quasi-totalité des ASC sont des femmes tandis que le rapport genre a été conservé dans le choix des RC. En général, les RC sont plus jeunes que les ASC. L'âge médian est respectivement 32 et 37 ans chez les RC et les ASC.

- Les ICP, superviseurs des ASBC : Tous les ICP (6) des zones expérimentales ont subi des entretiens approfondis au post-test.

- L'évaluation auprès de la population cible a été faite à partir de méthodes quantitatives et qualitatives:

* Pour les méthodes quantitatives, un échantillon, représentatif de femmes âgées de 15 à 49 ans et d'hommes âgés de 15 à 69 ans issus des deux zones expérimentales et de la zone de comparaison, a été interviewé lors des enquêtes CAP, avant et après les interventions communautaires. Ainsi deux échantillons de 1650 femmes et de 1500 hommes, également répartis entre les trois zones d'étude, ont été interviewés à l'enquête CAP de base. Lors de l'enquête CAP finale, ces échantillons ont été de 1572 femmes et de 1458 hommes. Ces échantillons de population correspondent aux tailles minimales ${ }^{11}$ d'échantillons nécessaires pour observer une augmentation statistiquement significative de la prévalence contraceptive par groupe de population cible.

* Pour les méthodes qualitatives, un échantillon de 69 hommes et femmes leaders communautaires (chefs de villages, présidents de communautés rurales, présidents et membres de comité de santé, les représentants des associations de femmes et de

\footnotetext{
${ }^{11}$ A partir du taux de prévalence contraceptive de la zone expérimentale trouvé à l'enquête CAP de base, il est calculé, pour l'enquête CAP finale, la taille d'échantillon minimale pour obtenir une augmentation de 5\% sur ce taux à un niveau de confiance $(1-\alpha)=95 \%$ et une puissance de test $(1-\beta)=80 \%$. Cette taille d'échantillon est considérée pour chacune des trois zones d'étude. La taille de l'échantillon total est la somme des échantillons enquêtés au niveau des trois zones d'étude.
} 
jeunes, et notables religieux) a été recruté pour organiser 8 groupes de discussions dirigées dans les deux zones où les interventions communautaires ont été expérimentées. Cette étude qualitative n'a été menée que dans le cadre des évaluations post-test. La base des données qualitatives est composée des groupes de discussions dirigées (GDD) et des entretiens individuels approfondis.

\section{Sources, collecte et analyse des données}

\section{- Sources et collecte des données}

Plusieurs sources de données sont utilisées pour déterminer l'efficacité, l'acceptabilité et la faisabilité des modèles communautaires expérimentés : les enquêtes sur les Connaissances, Attitudes et Pratiques (CAP), les Groupes de Discussions Dirigées (GDD) et les entretiens approfondis, l'interview des ASBC, les rapports mensuels d'activités et la documentation des coûts des interventions communautaires.

* Les enquêtes CAP : Deux enquêtes CAP ont été réalisées, avant et après l'intervention SBC. Ces enquêtes ont pour but de déterminer les connaissances, attitudes et pratiques des populations-cibles en matière de SR. Elles ont été menées auprès d'échantillons de femmes (15-49) et d'hommes (15-69) en âge de procréer des sites du projet. Elles ont été menées par deux équipes de 18 enquêtrices et 15 enquêteurs professionnels. Au préalable, ces agents de collecte ont reçu une orientation appropriée de trois jours pour se familiariser avec les instruments de collecte de données. Les enquêtrices ont fait l'interview des femmes et les enquêteurs celle des hommes. La durée des enquêtes CAP, au pré-test comme au post-test, a été la même : 3 semaines pour les femmes et les hommes.

* Les Groupes de Discussions Dirigées (GDD) et les entretiens approfondis: Ils ont été réalisés seulement auprès de personnes-ressources des deux zones expérimentales abritant les modèles communautaires. Plusieurs catégories d'informateurs ont été mises à contribution : les infirmiers-chefs de poste (ICP), les ASBC, les leaders communautaires (chefs de villages, autorités religieuses, présidents de communautés rurales, présidents ou membres de comités de santé, représentants des associations de femmes et de jeunes). Les GDD et les entretiens approfondis ont permis d'obtenir des données qualitatives qui permettent d'apprécier l'acceptabilité et la faisabilité des modèles communautaires mis en œuvre. Ce volet qualitatif a fait l'objet d'un rapport spécifique plus détaillé.

* L'interview des ASBC : Elle permet d'apprécier la faisabilité des modèles communautaires expérimentés. Dans ce sens un questionnaire a été administré aux ASC et aux RC pour évaluer leur appréciation sur la formation reçue, les tâches qui leur étaient assignées, le système de supervision de leurs activités, le taux d'occupation et le système de motivation instauré. Par ailleurs, les connaissances et attitudes des ASBC vis-à-vis des méthodes contraceptives promues dans le cadre de l'expérimentation, et leurs connaissances sur la SR des adolescents de leurs zones de responsabilité ont également été évaluées. L'interview des ASBC a été faite en trois jours par deux enquêteurs de l'enquête CAP finale.

* Rapports mensuels d'activités : Dans le cadre de l'intervention, chaque RC et ASC a reçu un cahier de vente, un cahier de référence et des fiches pictographiques pour faire la synthèse des activités réalisées au cours de la période bimestrielle. La fiche 
pictographique permet à chaque RC ou ASC de tenir les statistiques des activités IEC (causeries et visites à domicile), des ventes, des clientes servies et des références. La collecte des données des rapports mensuels d'activités a duré les 12 mois de la période d'expérimentation du projet et a été assurée par les ASC et RC eux-mêmes. La synthèse de ces rapports d'activités a été faite par les ASC et RC sous la supervision technique des ICP. L'analyse de ces différents rapports d'activités permet d'évaluer la performance des RC et des ASC.

* Documentation des coûts des interventions communautaires : Les données sur les coûts des différentes activités d'intervention communautaires ont été documentées auprès de MSH chargé de la réalisation de ce volet du projet. Les informations ont été collectées sur les coûts de conception et de mise en œuvre des interventions mais aussi sur les coûts supplémentaires de prestations de services. Le coordinateur de recherche a assure la collecte de ces données pendant la période de mise œuvre du projet. Ces statistiques ont été utilisées pour déterminer le coût global de chaque modèle communautaire et apprécier sa rentabilité. L'analyse des coûts des modèles communautaires expérimentés a également fait l'objet d'un rapport spécifique.

\section{- Traitement et analyse des données}

Les données des enquêtes CAP et des interviews des ASBC sont saisies avec le logiciel EPIDATA. La saisie des données a été assurée par deux équipes de quatre agents de saisie professionnels. Ces deux équipes ont travaillé de façon alternée chacune pendant une durée maximale de quatre heures par jour. Les agents de saisie ont reçu au préalable une orientation de trois jours pour se familiariser avec les questionnaires et les masques de saisie et faire des tests pratiques. Ils ont travaillé quotidiennement sous la supervision de l'assistant de recherche. La saisie des données a duré trois semaines. Ces données ont été saisies sous EPIDATA. Le traitement et l'analyse de ces données saisies ont été fait sous 'SPSS for Windows'. Le z-test a été utilisé pour tester les différences de niveau des indicateurs au pré et au post-test, du fait de la grande taille des échantillons. Par contre pour les indicateurs évalués au post-test seulement, l'appréciation des différences de niveaux selon les zones a été faite à partir du Chi-square pour les proportions simples et à l'analyse de la variance (ANOVA) pour les moyennes.

\section{Qualité des données et limites}

Pour assurer la qualité de la collecte des données, plusieurs dispositions pratiques ont été prises. Ces dispositions incluent :

- la révision des outils de collecte pour tenir compte uniquement des indicateurs pertinents pour l'évaluation des interventions communautaires ;

- le recrutement et la formation du personnel de collecte des données ;

- la reproduction des questionnaires ;

- l'information des autorités administratives et communautaires sur les objectifs des phases de collecte de données ;

- l'orientation des enquêtrices et enquêteurs sur le terrain ;

- la supervision quotidienne des enquêtrices et enquêteurs durant leur première semaine de collecte ;

- l'organisation après le terrain de réunions regroupant les agents de collecte, le chef d'équipe et l'équipe de recherche ;

- la documentation et le partage des solutions adoptées pour corriger les erreurs commises par les agents de collecte ; et 
- la correction des réponses erronées.

En général, la lecture du document de présentation de l'étude a suffi pour avoir le consentement des populations et administrer l'interview. La note d'information envoyée par le Médecin-Chef de District aux autorités administratives (préfet, sous-préfets, présidents de communautés rurales et chefs de villages ou de quartiers) a contribué à intéresser les populations à l'étude. Un seul cas de refus a été enregistré par les enquêtrices au niveau de la zone de comparaison et tient à un non-intérêt pour l'étude. Parmi les facteurs importants pouvant affecter les données collectées l'on peut citer :

- La différence entre les zones d'expérimentation et la zone de comparaison: Le choix des zones a plus répondu à des préoccupations d'ordre opérationnel du district qu'à des facteurs tenant compte de l'intervention. Bien que la zone de comparaison soit une zone rurale du même district, de part sa position géographique, elle est beaucoup plus urbanisée que les deux zones expérimentales.

- L'influence de la période de collecte : La période de collecte de l'enquête CAP Post-test a eu lieu à une période différente de celle du Pré-test. Elle a coïncidé avec la saison sèche correspondant à une période de fortes migrations de la population rurale vers les villes. Ces mouvements migratoires ont surtout été accentués par une mauvaise campagne agricole. Le fait que ces migrations ne concernent en général que les jeunes hommes a rendu difficile dans certains cas de figures, la constitution des échantillons d'hommes enquêtés au post-test. Dans ces cas de figures, le taux de sondage a été modifié pour obtenir la taille d'échantillon désirée. Cette situation a conduit à interroger un échantillon d'hommes plus âgés au post-test qu'au pré-test. Ainsi, les données collectées dans ce contexte peuvent être de nature à sous-estimer le niveau réel des indicateurs de SR.

\section{RESULTATS}

\section{Description des échantillons}

Des données qualitatives et quantitatives ont été utilisées pour évaluer les objectifs du projet. Les tableaux $2 \mathrm{a}$ et $2 \mathrm{~b}$ en annexe donnent les caractéristiques socio-démographiques des hommes et femmes interviewés au cours des deux enquêtes CAP dans les zones d'intervention du projet et dans la zone de comparaison.

Les échantillons de femmes enquêtées au pré-test (CAP pré-intervention) et au post-test (CAP post-intervention) présentent quelques spécificités selon les zones d'études. Du point de vue de l'âge, l'échantillon de femmes enquêtées au post-test dans la zone ASC est en moyenne plus jeune que celui enquêté au pré-test. Quant à la zone $\mathrm{RC}$, son échantillon posttest inclut une proportion plus élevée d'analphabètes. Dans les deux zones expérimentales, le niveau de fécondité des femmes enquêtées est plus bas qu'il ne l'était au pré-test. Mais l'âge moyen de leur dernier enfant, comme pour l'échantillon de femmes enquêtées dans la zone de comparaison est nettement plus élevé. Cette situation pourrait avoir une incidence négative sur le niveau de demande en PF notamment.

Les échantillons d'hommes interviewés lors des enquêtes CAP se caractérisent par une proportion plus élevée de personnes âgées au post-test qu'au pré-test. Cette différence d'âge est liée à la période de collecte des données pour les évaluations post-test. Pendant le post- 
test, la collecte a coïncidé avec la période de saison sèche pendant laquelle les zones rurales sont fortement marquées par des migrations saisonnières des jeunes hommes vers les zones urbaines. Cette différence d'âge, n'a aucun effet sur le niveau d'instruction des échantillons interrogés. Mais elle pourrait également avoir une incidence négative sur le niveau de demande en PF.

\section{Acceptabilité des modèles communautaires}

Elle a été évaluée en termes de perceptions de la communauté vis-à-vis des différents modèles de prestation de services, mais aussi de sa volonté de la communauté à utiliser ces services et à les recommander comme sources de services de SR. Elle a été mesurée à travers l'enquête CAP post-intervention et les groupes de discussions dirigées organisés avec les leaders communautaires et les représentants des associations de femmes et de jeunes.

Au niveau des enquêtes CAP, un ensemble de dix indicateurs a été utilisé pour apprécier le niveau d'acceptabilité du projet comme l'indique le tableau 3a. Chaque indicateur prend la valeur 1 si la réponse est 'OUI', et 0 si la réponse est 'NON'. Pour chaque personne interviewée, un indicateur d'acceptabilité est construit en additionnant l'ensemble des réponses à ces 10 indicateurs. Pour l'échantillon de population interviewé, on calcule un indice synthétique d'acceptabilité qui correspond au score moyen d'acceptabilité sur un maximum de 10 points possibles.

Tableau 3a : Indicateurs d'acceptabilité des modèles communautaires au niveau des femmes

\begin{tabular}{|c|c|c|c|}
\hline Indicateurs (pour chaque indicateur : oui $=1 ;$ non $=0$ ) & $\begin{array}{c}\text { Zone RC } \\
(n=422)\end{array}$ & $\begin{array}{c}\text { Zone ASC } \\
(n=449)\end{array}$ & $\begin{array}{l}\text { Test de } \\
\text { Fischer }\end{array}$ \\
\hline 1. Pense que la présence des ASBC est utile & 0.92 & 0.94 & .217 \\
\hline 2. Pense que les ASBC sont bien connus & 0.95 & 0.98 & .019 \\
\hline 3. Pense que les ASBC sont bien respectés** & 0.91 & 0.97 & .000 \\
\hline 4. Pense que les ASBC sont capables de garder le secret/les confidences** & 0.79 & 0.91 & .000 \\
\hline 5. Pense que les $\mathrm{ASBC}$ sont accessibles à tout le monde** & 0.83 & 0.92 & .000 \\
\hline 6. Pense que les ASBC doivent discuter de PF avec les jeunes & 0.81 & 0.75 & .024 \\
\hline $\begin{array}{l}\text { 7. Pense que les ASBC doivent discuter de PF avec les personnes non } \\
\text { mariées }\end{array}$ & 0.78 & 0.74 & .081 \\
\hline 8. Pense que l'accessibilité des services de santé s'est améliorée* & 0.83 & 0.90 & .004 \\
\hline $\begin{array}{l}\text { 9. Pense que les activités des ASBC doivent être poursuivies dans le } \\
\text { village }\end{array}$ & 0.91 & 0.94 & .062 \\
\hline 10. A déjà recommandé l'ASBC à un(e) ami(e) & 0.43 & 0.47 & .140 \\
\hline Indice synthétique d'acceptabilité & 8.2 & 8.5 & $\begin{array}{c}\text { Anova } \\
.011\end{array}$ \\
\hline
\end{tabular}

Source : CAP Femmes post-intervention, $2003 ; * * \mathrm{p}$ value $=.000 ; * \mathrm{p}$ value $<=.005$.

D'une manière générale, le niveau d'acceptabilité des deux modèles communautaires est similaire chez les femmes interrogées. En effet sur la base des 10 indicateurs pris simultanément, le score moyen d'acceptabilité est de 8.5 points sur une échelle de 10 points maximum possibles dans la zone ASC. Ce score est de 8.2 points sur 10 possibles dans la zone RC. La différence constatée n'est pas statistiquement significative. 


\begin{tabular}{|c|c|c|c|}
\hline Indicateurs (pour chaque indicateur: Oui $=1 ;$ Non $=0$ ) & $\begin{array}{c}\text { Zone RC } \\
(n=326)\end{array}$ & $\begin{array}{c}\text { Zone ASC } \\
(\mathbf{n}=\mathbf{3 0 4})\end{array}$ & $\begin{array}{l}\text { Test de } \\
\text { Fischer }\end{array}$ \\
\hline 1. Pense que la présence des ASBC est utile & 0.84 & 0.89 & .049 \\
\hline 2. Pense que les ASBC sont bien connus* & 0.92 & 0.98 & .002 \\
\hline 3. Pense que les ASBC sont bien respectés* & 0.89 & 0.97 & .000 \\
\hline 4. Pense que les ASBC sont capables de garder le secret/les confidences* & 0.83 & 0.89 & .016 \\
\hline 5. Pense que les ASBC sont accessibles à tout le monde* & 0.86 & 0.92 & .017 \\
\hline 6. Pense que les ASBC doivent discuter de PF avec les jeunes & 0.87 & 0.91 & .069 \\
\hline $\begin{array}{l}\text { 7. Pense que les ASBC doivent discuter de PF avec les personnes non } \\
\text { mariées }\end{array}$ & 0.86 & 0.89 & .197 \\
\hline 8. Pense que l’accessibilité des services de santé s’est améliorée* & 0.81 & 0.91 & .000 \\
\hline 9. Pense que les activités des ASBC doivent être poursuivies dans le village & 0.89 & 0.93 & .043 \\
\hline 10. A déjà recommandé l'ASBC à un(e) ami(e) & 0.50 & 0.45 & .094 \\
\hline Indice Valeur moyenne de l'indice* & 8.3 & 8.7 & $\begin{array}{c}\text { Anova } \\
.005 \\
\end{array}$ \\
\hline
\end{tabular}

Source : CAP Hommes post-intervention, $2003 ; * *$ p value $=.000 ;{ }^{*}$ p value $<=.005$.

Par contre, les résultats du tableau $3 \mathrm{~b}$ montrent que le niveau d'acceptabilité du modèle ASC est plus élevé que celui du modèle RC pour les hommes interrogés. L'indice synthétique d'acceptabilité est de 8.7 dans la zone ASC contre 8.3 dans la zone RC. Cette différence est statistiquement significative à $5 \%$.

Ces résultats montrent que, les hommes et les femmes ont le même niveau d'appréciation du modèle ASC. Par contre, le modèle communautaire avec RC est moins bien apprécié par les hommes que par les femmes. Malgré cette différence d'appréciation, le niveau d'acceptabilité du projet est, de façon générale, assez élevé. Ce résultat est confirmé au niveau des enquêtes qualitatives.

Au niveau des GDD et des entretiens approfondis, les critères d'appréciation utilisés sont la connaissance du projet SBC par les membres de la communauté, leurs perceptions des activités mises en œuvre et l'utilisation des services offerts par les ASBC.

Les rôles de l'ASBC tels qu'évoqués par les leaders et les membres de la communauté lors des GDD sont ceux d'éducateur, de conseiller et de promoteur en matière de $\mathrm{PF}$, de lutte contre les IST/SIDA et de santé de la reproduction. Les propos suivants d'une présidente d'un comité de santé de la zone ASC résument cette perception :

Quelle que soit la zone d'expérimentation, les services offerts par le projet SBC sont perçus comme permettant de renforcer la capacité des femmes dans la prévention et la prise en charge de la santé de la reproduction, et de soulager les hommes quant aux dépenses en matière de santé. Cette appellation descriptive du projet SBC dans un village de la zone RC est assez illustrative : "Dooleel Jigeen gni, Noppal goor gni » (signifiant 'Soutenir les femmes et soulager les hommes'). Dans leur quasi-totalité, les hommes et les femmes interrogées souhaitent la continuité du projet. Pour ces gens, les avantages liés au projet incluent : 
- l'accès facile aux informations et services de santé (PF, CPN, éducation sexuelle) ;

- l'accès facile aux médicaments ;

- l'acquisition de beaucoup de connaissances sur la santé ;

- les changements de mentalité vis-à-vis de la PF et de la CPN.

Les raisons avancées par ceux qui ne recommanderaient pas les ASBC incluent :

- la préférence du Poste de santé pour les consultations ;

- le fait que l'ASBC n'est pas qualifié ;

- le fait que l'ASBC n'habite pas dans le village.

\section{Faisabilité des modèles expérimentés}

Elle a été mesurée en termes de capacité des ASBC à remplir les tâches qui leur sont confiées et leur disponibilité constante à fournir ces services. Ces mesures ont été effectuées à partir des rapports d'activités, d'entretiens approfondis avec un échantillon de RC et d'ASC et à partir d'une analyse des coûts de chaque intervention.

Pendant la période de mise en œuvre du projet, les 10 tâches suivantes étaient assignées aux ASBC:

1. Exécuter des visites à domicile dans les ménages de leur zone de responsabilité ;

2. Animer des causeries éducatives pour la promotion de la santé de la communauté ;

3. Informer sur les différentes méthodes contraceptives ;

4. Mener des entretiens individuels et conseiller sur les différents aspects de la santé de la reproduction ( IST, CPN, accouchement assisté, AME, vaccination, pesée, maladies diarrhéiques et paludisme).

5. Disposer régulièrement de produits $\mathrm{SBC}$ en quantité suffisante ;

6. Assurer la vente des produits SBC;

7. Assurer le suivi des clients et référer au besoin au Poste de santé ;

8. Tenir correctement et au jour le jour les différents outils de gestion ;

9. Etablir et envoyer à l'ICP les rapports mensuels d'activités ;

10. Participer aux réunions de coordination de la zone.

Ces tâches ont généralement été exécutées comme l'indique le tableau 4 sur les rapports d'activités des ASBC.

Tableau 4: Nombre d'activités réalisées par tous les ASBC au cours des 10 premiers mois du projet

\begin{tabular}{|c|c|c|c|c|c|c|c|c|c|c|}
\hline & \multicolumn{2}{|c|}{ Causeries } & \multicolumn{2}{|c|}{ Visites à domicile } & \multicolumn{2}{|c|}{ Références } & \multicolumn{2}{|c|}{$\begin{array}{c}\text { Clients servis en } \\
\text { produits PF }\end{array}$} & \multicolumn{2}{|c|}{$\begin{array}{c}\text { Clients servis en } \\
\text { médicaments }\end{array}$} \\
\hline & RC & ASC & RC & ASC & RC & ASC & RC & $\mathrm{ASC}$ & RC & ASC \\
\hline Total selon le type de service & 550 & 507 & 347 & 712 & 653 & 469 & 258 & 191 & 3335 & 2553 \\
\hline Nombre de mois & 10 & 10 & 10 & 10 & 10 & 10 & 10 & 10 & 10 & 10 \\
\hline Nombre d'ASBC & $15^{*}$ & $15^{*}$ & 30 & 30 & 30 & 30 & 30 & 30 & 30 & 30 \\
\hline Dénominateur & 150 & 150 & 300 & 300 & 300 & 300 & 300 & 300 & 300 & 300 \\
\hline Nombre moyen par ASBC et par mois & 3.67 & 3.38 & 1.16 & 2.37 & 2.18 & 1.56 & 0.86 & 0.64 & 11.12 & 8.51 \\
\hline
\end{tabular}

\footnotetext{
* Le nombre de causeries effectuées est comptabilisé pour le binôme qui les co-anime .
} 
Tous les deux mois, chaque binôme d'ASBC devait déposer auprès de l'ICP un rapport d'activités. Le tableau 4 donne pour l'ensemble des 30 RC et 30 ASC les statistiques des services réalisés au cours des 10 premiers mois d'intervention du projet. Pour les deux derniers mois du projet, ces statistiques étaient incomplètes : seuls les ASC ont déposé leur rapport d'activités. Cependant l'exécution de cette tâche n'a pas été toujours facile. Il a fallu une orientation des ASBC pour le remplissage des fiches pictographiques et un suivi hebdomadaire effectué par le coordonnateur du volet Recherche pour les assister dans cette tâche. Les résultats des interviews des ASBC révèlent que 6 ASC sur 10 et 4 RC sur 10 éprouvaient des difficultés dans le remplissage de ces fiches. Pour remédier à cette insuffisance, ils ont souvent eu recours à l'assistance d'une tierce personne instruite. Cette situation est en partie liée au pourcentage élevé d'analphabètes parmi les ASBC $\quad(44 \%$ des ASC et $30 \%$ des RC).

Activités IEC : L'examen des rapports mensuels d'activités des ASBC montre que le binôme de RC a effectué en moyenne 4 causeries éducatives par mois contre 3 pour le binôme d'ASC. Les rapports d'activités montrent également que ces activités IEC ont porté sur les quatre composantes de la SR. Mais il est paradoxal de noter que l'ASC qui n'a pas l'obligation d'effectuer des visites à domicile a eu en moyenne à en réaliser 2 fois plus qu'un $\mathrm{RC}$ par mois. Ce résultat pourrait expliquer pourquoi l'ASC a effectué moins de causeries éducatives que le RC. Ces VAD n'ont également connu leur niveau de croisière qu'après le recyclage des ASBC.

Références : Au chapitre des références, la performance enregistrée en zone RC est meilleure qu'en zone ASC. En moyenne, chaque RC a référé 2.18 clients contre 1.56 pour l'ASC. Mais d'une manière générale, l'évolution des séries chronologiques sur le graphique 1 révèle une nette augmentation des références au fil du temps.

\section{Graphique 1: Evolution du nombre d'activités des ASBC}

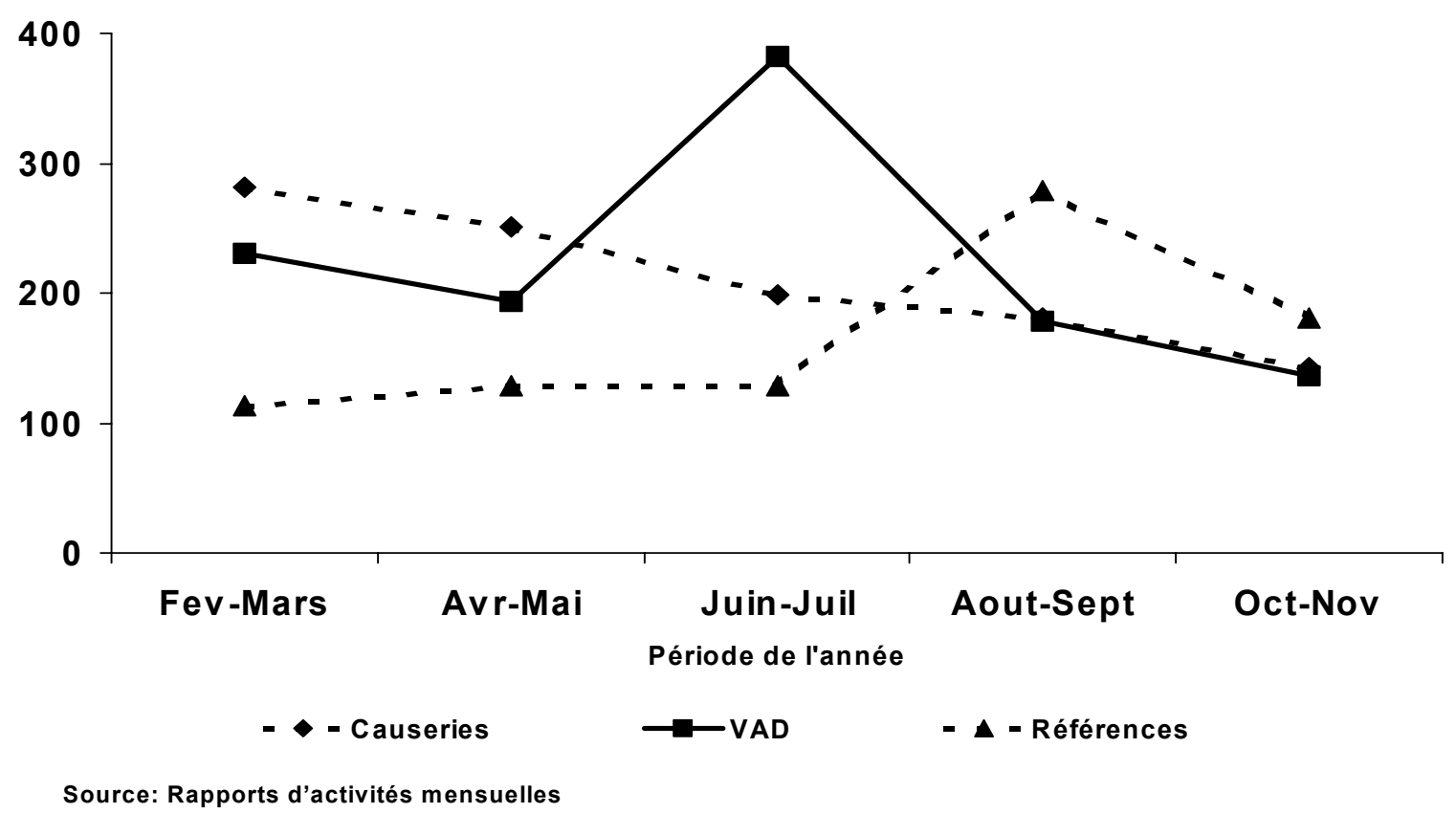


Vente des produits $S B C$ : L'examen du tableau 4 montre la faiblesse notaire du nombre mensuel de clientes servis en produits PF : en moyenne, chaque RC ou ASC a servi moins d'une cliente PF par mois. Par contre les données révèlent qu'en moyenne chaque mois le RC a servi davantage de clientes en médicaments que l'ASC: en moyenne 11.12 clientes pour le RC contre 8.51 pour l'ASC.

De manière générale, les performances des ASBC telles qu'elles ressortent des rapports d'activités sont faibles. En l'absence de sessions de recyclage, les activités IEC suivent une tendance à la baisse au fil du temps.

Disponibilité des produits $S B C$ : Le graphique 2 montre que le système de réapprovisionnement des $\mathrm{ASBC}$ en produits a connu des problèmes. Pour chaque produit considéré, des ruptures de stock existent au niveau de certains des ASBC interrogés. Il ressort également des données qu'aucun ASBC interrogé ne disposait de la totalité des huit produits listés. Sur ces 8 produits du projet, au plus 7 en étaient disponibles au niveau de $18 \mathrm{RC}$ et de 10 ASC. A l'inverse, le nombre minimum de produits disponibles au moment de l'enquête était de 1 chez les ASC et de 2 chez les RC.

De même que pour l'acceptabilité des modèles, on peut construire un indice synthétique sur la capacité des ASBC à réaliser les tâches qui leur étaient assignées ${ }^{12}$. Cet indice synthétique est de 8.4 pour les RC et 8.5 pour les ASC sur un score maximum de 10 points possibles. Sur la base de ces résultats, l'on peut dire que la capacité de réalisation des tâches est à un niveau assez élevé et similaire pour les deux modèles.

La faisabilité des deux modèles communautaires a également été appréciée à partir des opinions des ASBC sur la formation reçue, la supervision, la motivation et la pérennité des activités SBC.

\footnotetext{
${ }^{12}$ Les indicateurs considérés sont : (1) ont réalisé des activités IEC ; (2) ont vendu des produits SBC ; (3) ont référé des clients ; (4) ont utilisé les affiches ; (5) ont utilisé le mannequin ; (6) ont utilisé les cartes IST ; (7) ont utilisé l'échantillon de produits contraceptifs ; (8) ont utilisé les dépliants ; (9) n'ont jamais connu de rupture de stock de produits SBC; (10) n'ont pas eu des problèmes relatifs au remplissage des fiches pictographiques.
} 


\section{Graphique 2: Pourcentage d'ASBC qui ne disposaient pas de produits de PF et de médicaments au moment de l'enquête}

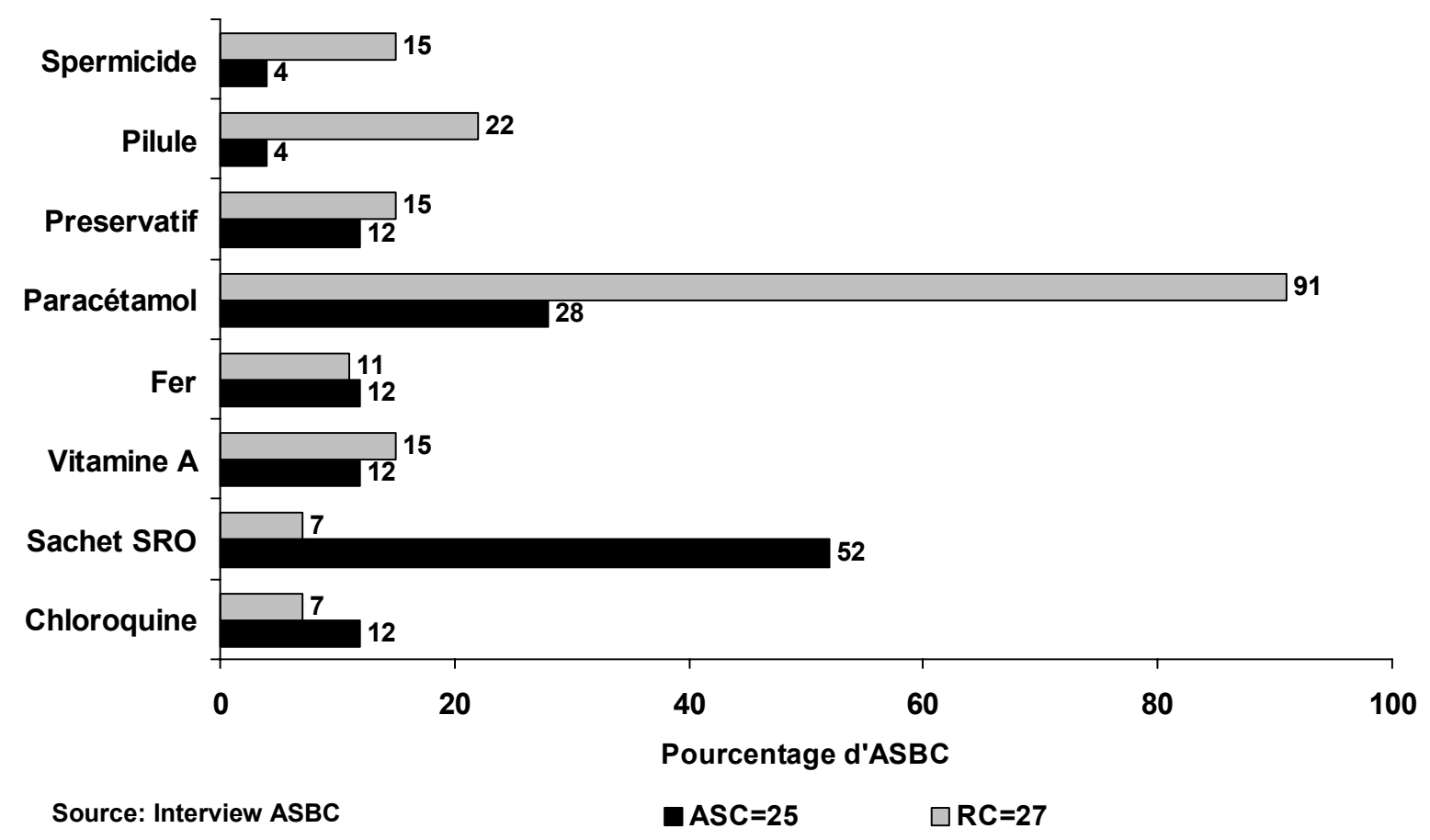

Formation reçue: L'analyse des opinions des ASBC sur la formation reçue donne un indice de satisfaction ${ }^{13}$ identique pour les deux modèles égal à 6 points sur 10 possibles. Les résultats des interviews montrent que près de 4 ASBC sur 5 sont satisfaits du contenu de la formation qu'ils ont reçue. Mais ils sont moins satisfaits de la durée de la formation de base et du nombre de sessions de recyclage effectuées.

\footnotetext{
${ }^{13}$ L'indice de satisfaction a été construit par rapport à la durée de la formation, au contenu de la formation et au nombre de sessions de recyclage effectuées suivant la même méthodologie utilisée précédemment.
} 


\section{Graphique 3: Répartition des ASBC selon leur opinion sur la formation reçue}

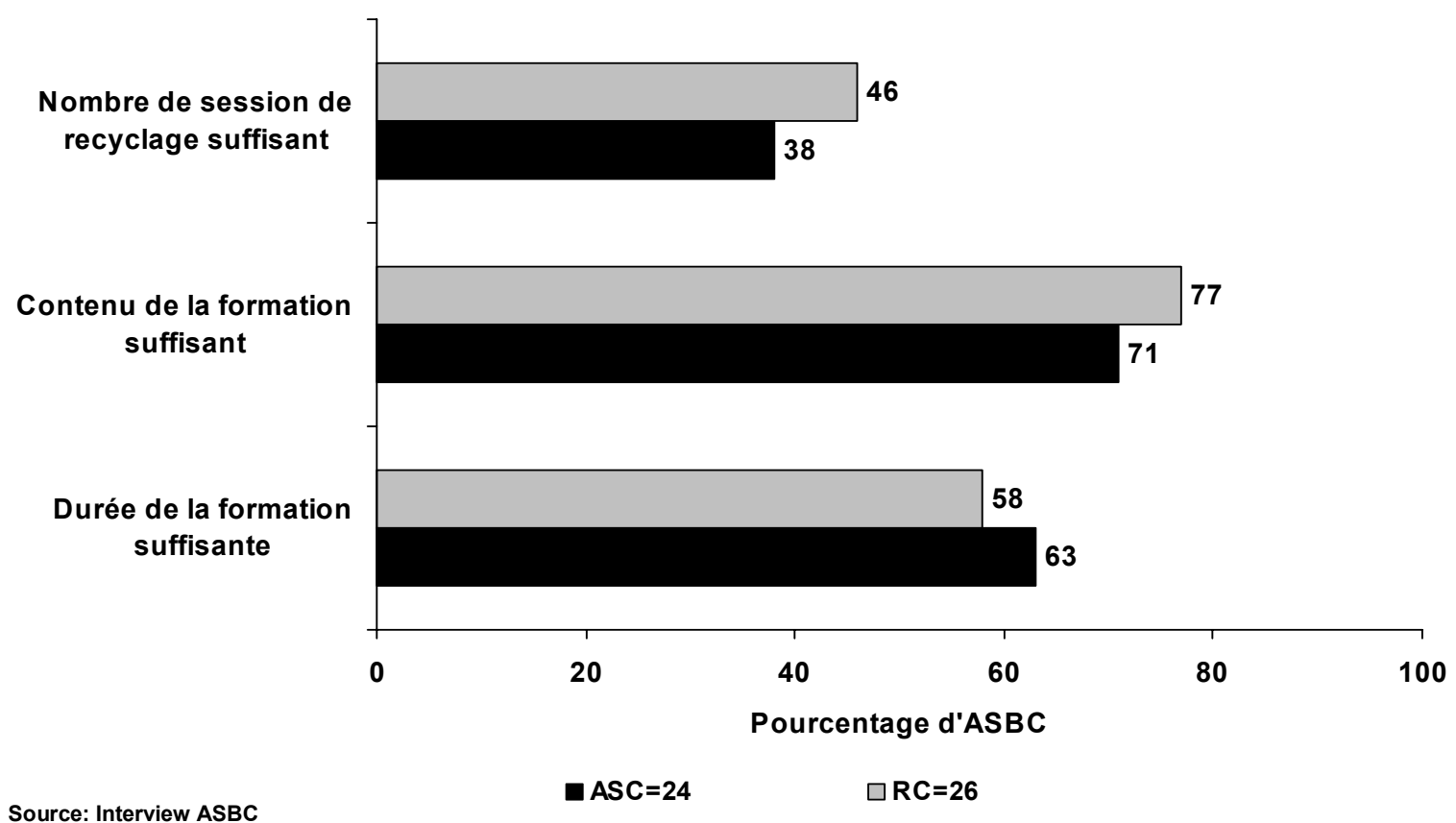

La durée de la formation de base, 12 jours, a été jugée insuffisante par 4 ASBC sur 10. Quant au nombre de sessions de recyclage, plus de la moitié des ASBC l'ont jugé insuffisant. En réalité, une seule session de recyclage a été réalisée contre quatre de prévues. La principale suggestion faite par les ASBC pour l'amélioration de leur formation est un recyclage périodique plus fréquent : au moins une session tous les 6 mois.

Supervision: La plupart des ASBC ont déclaré avoir reçu au moins une visite de la part de leur superviseur au cours des 12 mois d'expérimentation (96\% des ASC et $82 \%$ des RC). Un ASC et $5 \mathrm{RC}$ ont déclaré n'avoir reçu aucune visite de supervision. Le nombre médian des visites supervision est égal à 2 . Toutefois, la majorité des 52 ASBC interviewés estime que les visites de supervision doivent être plus fréquentes. Ce sentiment est partagé par $81 \%$ des ASC et $71 \%$ des RC.

La stratégie d'expérimentation des approches communautaires prévoyait une supervision mensuelle des ICP au cours des 6 premiers mois de l'intervention et une supervision trimestrielle au cours du second semestre. Sur le terrain, ce rythme de supervision n'a pas été respecté. Sa réalisation s'est heurtée à des difficultés liées à la charge de travail élevée des ICP qui rendait difficile l'organisation de supervisions spécifiques aux activités SBC et à l'insuffisance de ressources financières pour la prise en charge de ces visites de supervision. Les rares visites ont coïncidé avec les tournées évaluatives organisées conjointement par les membres du comité technique et l'équipe-cadre du district. D'autres contacts avec les ASBC ont également eu lieu à l'occasion des stratégies avancées des ICP.

Motivation: Le projet a octroyé à chaque binôme de RC, un cheval et une charrette pour leurs moyens de déplacement. Chaque RC était autorisé à garder $75 \%$ des bénéfices tirés de la vente des produits SBC. En outre, les RC étaient autorisés à utiliser le cheval et la charrette pour d'autres activités génératrices de revenus : transport de personnes ou de marchandises, travaux champêtres, évacuation de malades vers les postes ou le centre de santé. Pour les 
ASC, en plus du traitement spécifique accordé par le comité de santé de la Case de santé, l'ASC était autorisé à garder $75 \%$ des bénéfices tirés de la vente des produits SBC.

Cependant, à la question 'Recevez-vous une motivation quelconque en compensation de votre travail ?', une faible proportion des ASBC (17\% des RC et $32 \%$ des ASC) a répondu par l'affirmative. Quelle que soit la nature de cette motivation, la plupart de ceux qui l'ont reçue trouvent qu'elle n'a pas satisfait à leurs attentes.

Pérennisation: Dans la pratique, les RC et les ASC ont consacré, respectivement, $20 \%$ et 34 $\%$ de leur temps libre aux activités SBC. Aussi, tous les ASBC interrogés ont manifesté le désir de continuer à servir comme tels. Mais selon les ASBC eux-mêmes, les conditions préalables à cette continuation seraient une motivation financière substantielle, l'amélioration des moyens de déplacement, l'augmentation de la dotation en produits SBC et des sessions de recyclage périodiques.

\section{Efficacité des modèles expérimentés}

L'efficacité de chaque modèle communautaire est mesurée sur la base du taux de couverture de la population-cible de chaque modèle, ainsi que des niveaux de connaissance et d'utilisation des services de santé de la reproduction au sein des communautés ciblées. Ces indicateurs ont été estimés à partir des données collectées des enquêtes CAP pré et postintervention au niveau des populations des trois zones d'étude.

\section{- Couverture géographique des modèles RC et ASC}

Dans la zone RC, le taux de couverture a été calculé sur la base du nombre de villages effectivement visités par le RC par rapport au nombre total de villages dans son aire de responsabilité. Par contre, dans la zone ASC, ce taux est calculé sur la base du nombre de villages qui ont effectivement fréquenté la Case de santé par rapport au nombre total de villages satellites de la Case de santé. Sur cette base, le taux de couverture est évalué à $90 \%$ dans la zone RC contre $94 \%$ dans la zone ASC.

\section{- Couverture de la population par les modèles RC et ASC}

Pour les besoins d'analyse, l'on a adopté la définition opérationnelle qu'une personne était couverte par le modèle si :

- $\quad$ elle a déjà assisté à une causerie éducative ; ou

- $\quad$ elle a reçu une visite à domicile de l'ASBC; ou

- $\quad$ elle a rencontré l'ASBC chez ce dernier ou dans un autre endroit ; ou

- $\quad$ elle a déjà acheté un produit contraceptif auprès de l'ASBC; ou

- $\quad$ elle a déjà acheté un médicament auprès de l'ASBC ; ou

- $\quad$ elle a déjà été référée par l'ASBC.

Les données de l'enquête CAP post-test montrent que le taux de couverture est quasiment le même pour les deux modèles, les différences notées n'étant pas statistiquement significatives. Dans la zone RC, ce taux est de $64 \%$ pour les femmes et $46 \%$ pour les hommes. Dans la zone ASC, ces taux sont de $71 \%$ pour les femmes et $48 \%$ pour les hommes. Ces deux résultats confirment que le taux de couverture des deux modèles est le même, que ce soit en termes de villages ou de population couverts. 


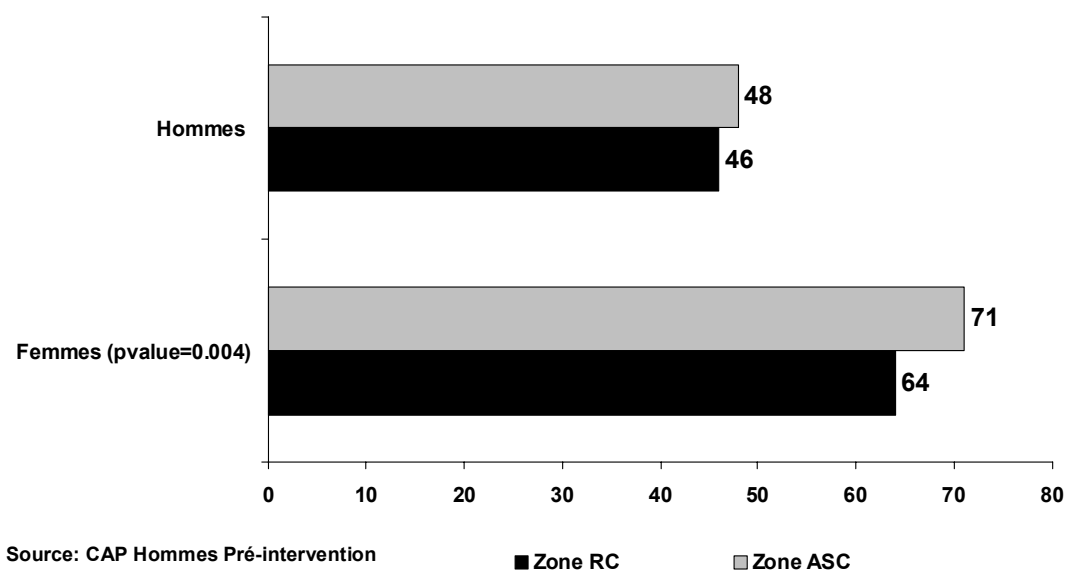

- Connaissance des services de SR

Pour chaque composante de la santé de la reproduction retenue, un indice synthétique a été construit à partir d'un certain nombre d'indicateurs de connaissances (voir tableaux 9, 10, 11 et 12 en Annexe). A partir de ces indices respectifs, un indice synthétique du niveau de connaissance des services de SR a été construit.

Tableau 5 : Indice synthétique du niveau de connaissance en SR

\begin{tabular}{|c|c|c|c|c|c|c|}
\hline \multirow[b]{2}{*}{ Indicateurs } & \multicolumn{3}{|c|}{ Pré-intervention } & \multicolumn{3}{|c|}{ Post-intervention } \\
\hline & $\begin{array}{c}\mathrm{RC} \\
(n=513)\end{array}$ & $\begin{array}{c}\text { ASC } \\
n=516)\end{array}$ & $\begin{array}{l}\text { COMP } \\
(n=480)\end{array}$ & $\begin{array}{c}\mathrm{RC} \\
(n=513)\end{array}$ & $\begin{array}{c}\text { ASC } \\
(n=511)\end{array}$ & $\begin{array}{l}\text { COMP } \\
(n=486)\end{array}$ \\
\hline 1. Indice connaissance en Planification Familiale & 0.477 & 0.434 & 0.591 & $0.663^{*}$ & $0.683^{*}$ & $0.726^{*}$ \\
\hline 2. Indice connaissance en VIH/SIDA & 0.482 & 0.474 & 0.540 & $0.568 *$ & $0.592 *$ & $0.662 *$ \\
\hline 3. Indice connaissance en Maternité à moindre risque & 0.665 & 0.694 & 0.706 & $0.675^{*}$ & 0.698 & 0.723 \\
\hline 4 - Indice connaissance en Survie de l'enfant & 0.686 & 0.753 & 0.816 & $0.786^{*}$ & 0.803 & $0.906^{*}$ \\
\hline Valeur de l'indice calculé sur les 4 items listés & 0.578 & 0.589 & 0.663 & $0.673 *$ & $0.694 *$ & $0.754 *$ \\
\hline
\end{tabular}

$* \mathrm{p}<.05$

L'hypothèse 1 postulait que les niveaux de connaissances seraient meilleurs à ceux qu'ils étaient avant l'intervention. Cette hypothèse n'est pas confirmée pour les 4 volets de la SR comme l'indique le tableau 5.

Bien que les niveaux de connaissance en matière de FP et de VIH/SIDA des femmes des zones où les deux models DBC aient été introduit ont augmenté de façon significative après les interventions, une augmentation similaire a été constatée au niveau de la zone de comparaison. On en conclut que les models DBC n'ont pas eu un effet sur le niveau général d'amélioration des connaissances. Une augmentation significative du niveau de connaissance en matière de maternité sans risque a été observée uniquement dans la zone RC ; et des augmentations significatives des connaissances en matière de survie de l'enfant ont été observé dans les deux zones RC et de comparaison.

Selon l'hypothèse 2, les niveaux de connaissances en SR devraient être plus élevés dans les villages où les interventions communautaires ont été introduites que dans les villages couverts par l'intervention qui porte uniquement sur le renforcement des formations sanitaires. Mais les données révèlent que le niveau de l'indice synthétique était déjà plus élevé dans la zone de comparaison que dans les zones expérimentales au début de l'intervention. Cette situation n'a pas changé au post-test. Dans ce cas, l'analyse a plutôt 
porté sur les accroissements de l'indicateur dans chaque modèle de prestation de services. Ainsi, les résultats montrent que les améliorations enregistrées au niveau de l'indice au posttest sont plus importantes dans les zones expérimentales ( 0.105 en zone ASC et 0.095 en zone RC) que dans la zone de comparaison (0.091). Ce qui confirme l'hypothèse.

L'hypothèse 2 est également confirmée pour le volet PF où les améliorations enregistrées au post-test sont plus importantes au niveau des zones expérimentales ( 0.249 en zone ASC, 0.186 en zone RC) qu'au niveau de la zone de comparaison (0.135). Par contre, les améliorations enregistrées sur les connaissances par rapport au VIH/SIDA et à la maternité à moindre risque sont plus importantes dans la zone de comparaison que dans les zones expérimentales. Les résultats sont plus mitigés pour le volet 'Survie de l'enfant'. L'hypothèse se confirme seulement dans la zone RC où l'amélioration ( 0.1$)$ est plus importante que celle enregistrée dans la zone de comparaison (0.09). Par contre, l'amélioration enregistrée par la zone ASC (0.05) est inférieure à celle de la zone de comparaison.

Quant à l'hypothèse 3, elle postulait que les niveaux de connaissances au post-test seraient meilleurs dans les zones d'intervention des relais communautaires que dans les zones des ASC. Cette hypothèse n'est pas confirmée par les données. L'indice synthétique est, au contraire, à un niveau légèrement supérieur dans les zones ASC aussi bien au pré-test qu'au post-test.

Cette situation se confirme pour les volets PF et IST/VIH/SIDA individuellement pris. Les améliorations enregistrées au post-test sont significatives et plus importantes dans les zones des ASC que dans les zones d'intervention des relais communautaires. Par contre, pour le volet Maternité à moindre risque, l'intervention dans la zone ASC n'a pas eu d'effet significatif. De même pour la Survie de l'enfant, seule la zone expérimentale avec RC a enregistré une amélioration significative. C'est au niveau de ce volet seulement que l'hypothèse 3 se confirme.

\section{- Attitudes vis-à-vis de la PF et des IST/VIH/SIDA}

L'étude n'avait pas formulé des hypothèses sur les attitudes des populations étudiées vis-à-vis de la PF et des IST/VIH/SIDA. Mais l'on pouvait s'attendre à ce qu'une amélioration des connaissances en services de SR en général et en PF et IST/VIH/SIDA en particulier soit accompagnée par des attitudes plus favorables vis-à-vis de ces deux composantes de la SR. Sur les mêmes principes méthodologiques, un indice synthétique a été construit pour les attitudes vis-à-vis de la PF et un autre pour les attitudes vis-à-vis des IST/VIH/SIDA (voir tableaux 13 et 14 en Annexe).

Des améliorations significatives des attitudes vis-à-vis de la planification familiale et par rapport à la discussion en publique du thème de VIH/SIDA ont été observées dans les deux zones DBC mais pas dans la zone de comparaison

Ainsi, dans les zones expérimentales avec RC et ASC, les niveaux atteints par l'indice synthétique sur les attitudes favorables à la PF sont supérieurs à ce qu'ils étaient au pré-test. Dans la zone de comparaison par contre, l'indice n'a pas connu d'amélioration. Par ailleurs, l'amélioration de l'indice enregistrée au niveau de la zone RC est plus importante que celle enregistrée dans la zone ASC.

Les niveaux de l'indice synthétique sur les attitudes vis-à-vis du VIH/SIDA sont meilleurs à ce qu'ils étaient avant les interventions. Les améliorations enregistrées au niveau des zones 
expérimentales avec RC et avec ASC sont plus importantes que celles enregistrées au niveau de la zone de comparaison. L'analyse selon la zone expérimentale montre que l'amélioration enregistrée au niveau de la zone RC est plus importante que celle de la zone ASC. De ces analyses, il ressort que les interventions communautaires ont également eu comme résultat la promotion d'attitudes favorables à la PF et à la prévention du VIH/SIDA.

\section{- Pratique de la PF}

La pratique de la PF est mesurée à travers l'utilisation d'une méthode contraceptive au cours des 12 derniers mois ayant précédé l'enquête et l'utilisation d'une méthode contraceptive au moment de l'enquête.

A l'instar de l'impact que les interventions ont eu au niveau des attitudes des populations étudiées vis-à-vis de la $\mathrm{PF}$, les niveaux de la pratique contraceptive ne diffèrent pas sensiblement de ce qu'ils étaient avant l'intervention. D'une zone expérimentale à l'autre, les améliorations enregistrées au niveau de la zone ASC sont plus importantes que celles

\section{Graphique 5: Pourcentage de femmes mariées qui ont utilisé une méthode contraceptive au cours des 12 derniers mois}

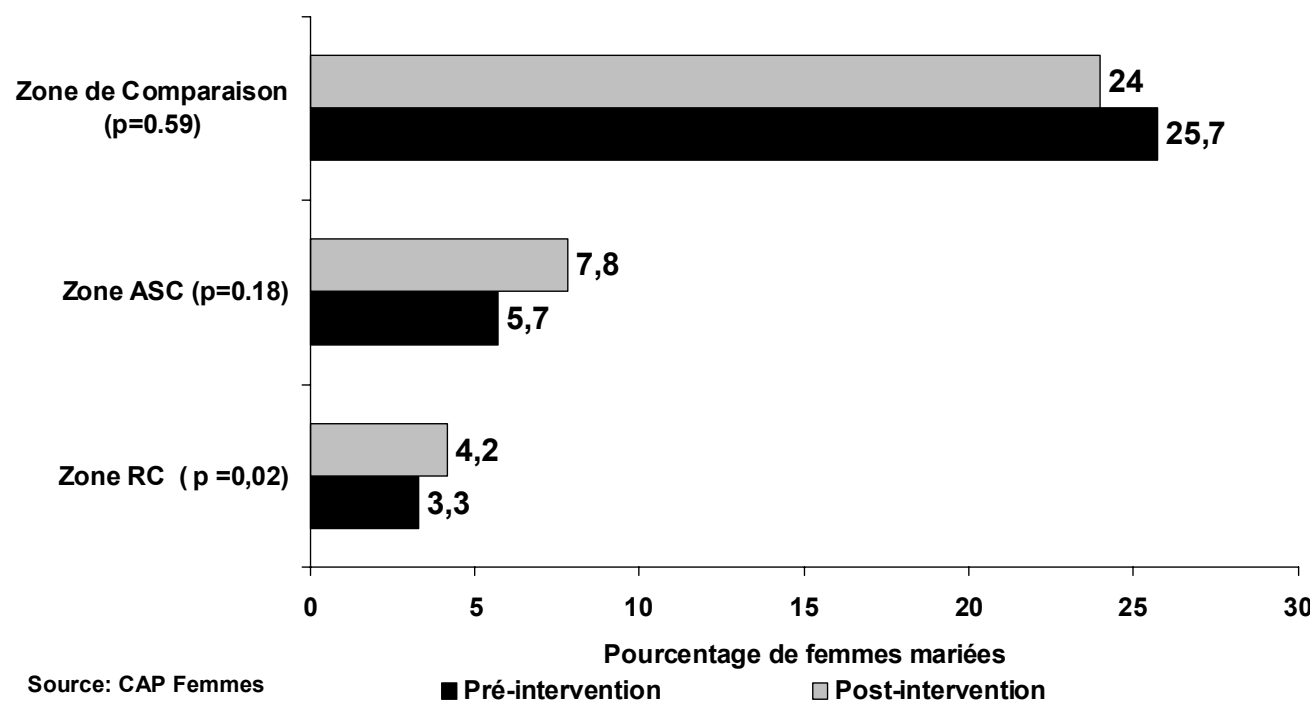

enregistrées au niveau de la zone RC.

Les proportions de femmes mariées qui ont utilisé une méthode contraceptive au cours des 12 derniers mois ayant précédé la collecte des données ont enregistré une augmentation après l'introduction des deux modèles. Mais ces augmentations étaient statistiquement significatives (à 5\% du niveau de signification) pour le modèle RC seulement (une augmentation de 3.3 à 4.2\%). Dans la zone de comparaison, il n'y a pas eu de changement ; en fait, une diminution de l'utilisation par les femmes a été observée dans la zone de comparaison (graphique 5). Ces résultats indiquent une stagnation ou une baisse dans l'utilisation de la planification familiale au niveau des deux modèles dans le district de Kébémer. Comme l'on pouvait s'y attendre, il n'y a pas eu de changement dans l'utilisation du condom par les hommes. L'utilisation du condom est beaucoup plus élevé par les hommes non-mariés que par les hommes mariés 
L'évaluation post-intervention s'est, par ailleurs, intéressée aux femmes qui n'étaient pas sous méthode au moment de l'enquête pour en savoir plus sur leurs intentions futures en matière de pratique contraceptive. Il en a résulté que, parmi ces femmes, des proportions élevées déclarent qu'elles n'ont pas l'intention d'utiliser une méthode contraceptive dans l'avenir : $67 \%$ en zone RC, $63 \%$ en zone ASC et $66 \%$ en zone de comparaison. Le tableau 15 en annexe présente les raisons principales avancées pour expliquer cette attitude selon la zone: (1) veut des enfants ; (2) est opposée à la PF ; (3) a des problèmes de santé; (4) effets secondaires; (5) est en ménopause; et (7) pas commode.

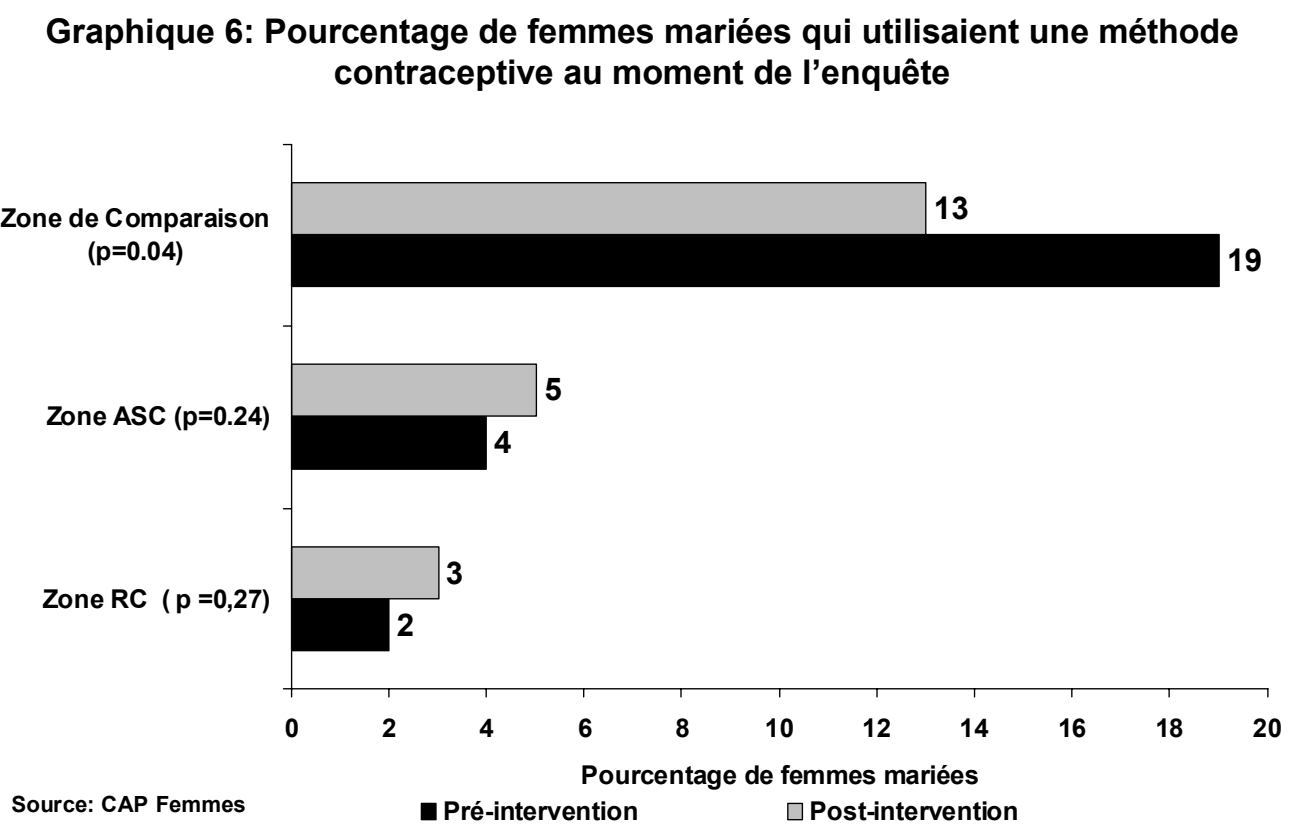

Il est intéressant de noter que les quatre premières raisons évoquées peuvent être résolues à travers une action programmatique. La première soulève le problème de l'argumentaire de la PF qui reste à être davantage étoffé. Cette situation a d'ailleurs été soulignée au niveau des Journées Nationales sur la SR organisées par le MSP avec l'appui de l'USAID/Sénégal en avril 2003.

Relativement aux hommes, le pourcentage de ceux qui ont utilisé le préservatif au cours des 12 derniers mois ayant précédé la collecte a été évaluée à l'enquête de base et à l'enquête finale.

Les niveaux atteints au niveau des zones expérimentales ne diffèrent pas sensiblement de ce qu'ils étaient avant l'intervention. Dans la zone RC, une timide évolution de l'indicateur a été enregistrée. Quant à la zone ASC, la tendance à la baisse qui est notée reflète quand même une spécificité de l'intervention dans cette zone. Les ASC sont principalement des femmes et elles ont souvent rapporté que les hommes éprouvent de la gêne à s'approvisionner en préservatif auprès d'elles. Cette situation pourrait expliquer la tendance observée dans la zone ASC quant à l'utilisation du préservatif par les hommes. 


\section{Graphique 7: Pourcentage d'hommes qui ont utilisé le préservatif au cours des 12 derniers mois}

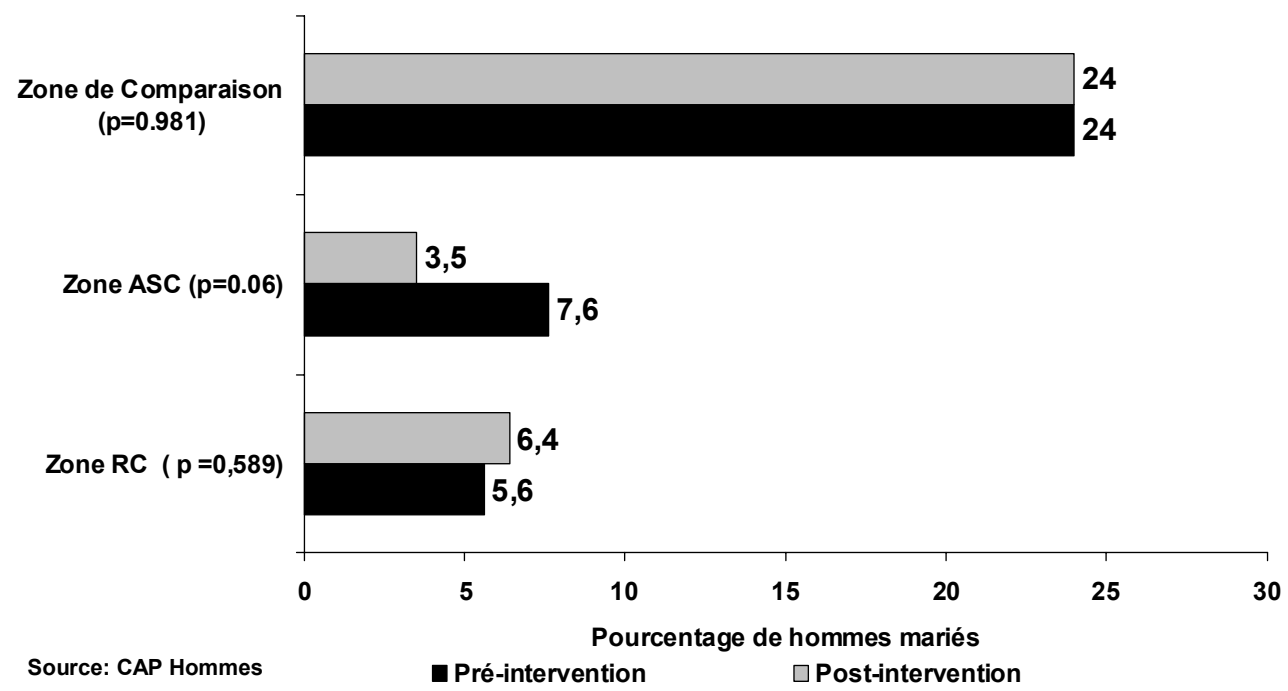

Par ailleurs, l'étude s'est intéressée à l'utilisation du préservatif par les hommes selon leur statut matrimonial. Les pourcentages respectifs d'hommes mariés et non mariés qui ont utilisé le préservatif au cours des 12 derniers mois ayant précédé la collecte des données ont été évalués avant et après l'intervention. De même, il a été évalué, dans le cadre de l'enquête post-intervention, les pourcentages respectifs d'hommes mariés et non mariés qui ont utilisé le préservatif lors de leur dernier rapport sexuel.

Graphique 8a: Pourcentage d'hommes mariés et non mariés qui ont déclaré avoir utilisé le préservatif au cours des 12 derniers mois

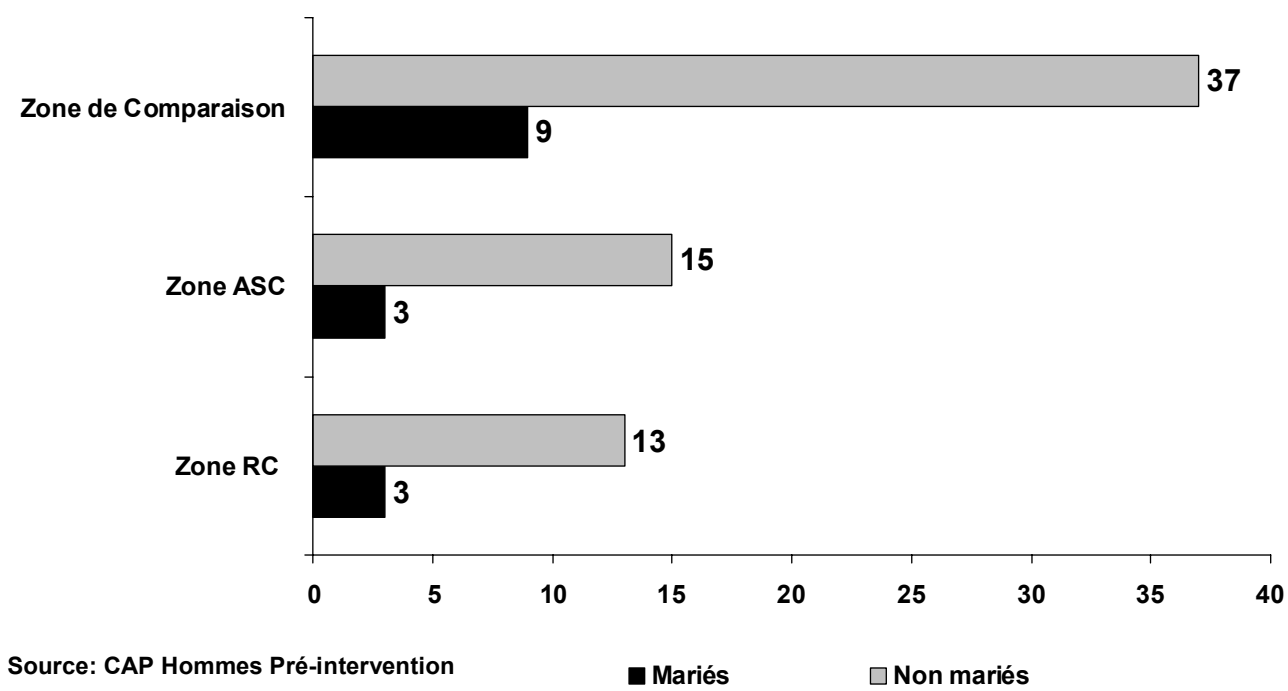

Il ressort des graphiques $8 \mathrm{a}$ et $8 \mathrm{~b}$ que, quelles que soient la période de collecte des données et la zone d'étude, l'usage du préservatif est plus effectif au niveau des hommes non mariés qu'au niveau des hommes mariés. En d'autres termes l'usage du préservatif est plus systématique en dehors que dans le cadre du mariage. 


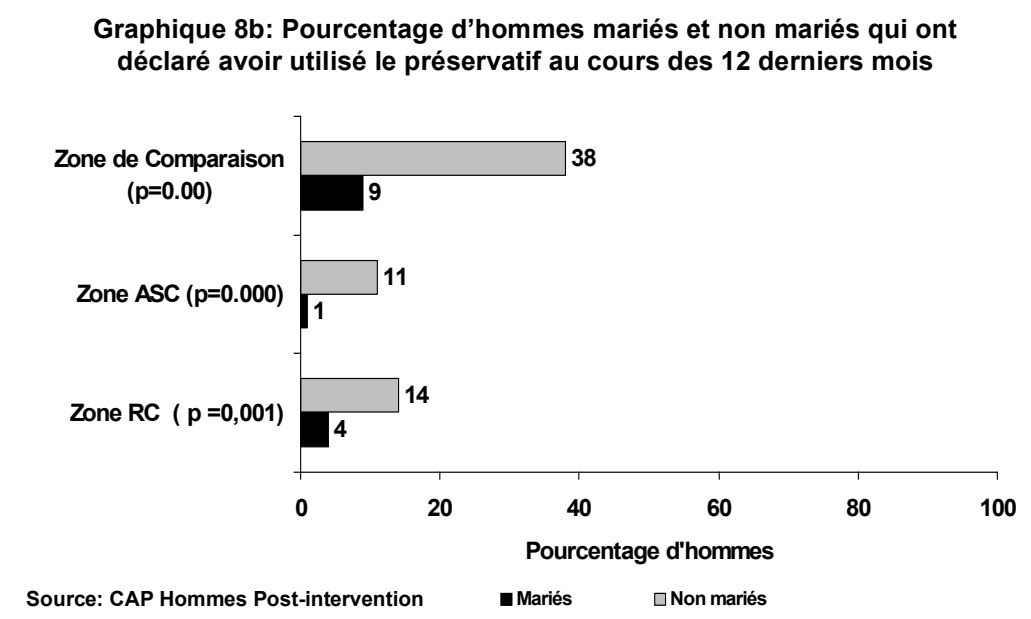

Cette situation est confirmée avec l'évaluation, dans le cadre de l'enquête post-intervention, des pourcentages respectifs d'hommes mariés et non mariés qui ont utilisé le préservatif lors de leur dernier rapport sexuel. Dans la zone RC, ils sont respectivement $6 \%$ et $40 \%, 2 \%$ et $24 \%$ dans la zone ASC, et $8 \%$ et $54 \%$ dans la zone de comparaison. Le faible usage du préservatif dans le cadre du mariage conforte encore la thèse de l'absence d'un argumentaire étoffé pour la pratique contraceptive.

\section{- Utilisation des services du programme SBC}

L'utilisation des services des ASBC peut être mesurée par l'importance de la population cible qui a

utilisé dans la période de l'enquête, ou a déjà utilisé au cours des 12 derniers mois qui ont précédé l'enquête les services de SR fournis par les modèles RC et ASC. Cette statistique est la même que le taux de couverture de la population par les modèles ASC et RC calculée en début de chapitre. Ces données indiquent que les hommes ont moins utilisé les services des ASBC que les femmes quelle que soit la zone d'intervention ASC ou RC. Dans les deux zones un peu moins de la moitié des hommes ont eu à les utiliser tandis que chez les femmes environ deux femmes sur trois ont recouru à ces services. L'analyse selon la zone d'intervention indique que l'utilisation des services SBC chez les hommes est la même quelle que soit la zone. Chez les femmes par contre on note une différence statistiquement significative dans l'utilisation des services SBC en faveur du modèle ASC : 64\% en zone RC contre $71 \%$ en zone ASC.

\section{Analyse des coûts}

L'analyse des coûts dans le cadre du projet SBC porte sur l'étude des coûts des ressources nécessaires pour réaliser le projet. Son importance réside dans les deux aspects essentiels suivants:

- Elle est la première étude du genre réalisée au Sénégal ;

- Elle fournit au Ministère de la Santé et de la Prévention, aux ONGs et autres Agences de Collaboration des informations de base utiles pour la réplication de l'approche SBC dans d'autres districts du pays. 


\section{Méthodologie}

\section{Description du modèle}

La présente étude d'analyse des coûts repose sur les postulats suivants :

- Le coût de chaque activité du processus de mise en œuvre de l'intervention SBC peut être évalué;

- Chaque spécificité ou élément de l'intervention SBC a également un coût que l'on peut évaluer ;

- En additionnant ces différents coûts, on peut déterminer les ressources nécessaires à la mise en œuvre de l'intervention SBC.

Le modèle d'analyse des coûts présenté dans ce rapport comprend deux parties. La première partie correspond à la phase des activités préparatoires et la seconde partie à la phase de fonctionnement ou mise en œuvre des activités modèles SBC expérimentés.

Les activités préparatoires des interventions SBC se sont étalées sur 8 mois et ont consisté : en des concertations au niveau central, au niveau du district et au niveau de la communauté pour présenter le projet, à des séances de formation des ICP/superviseurs et des agents SBC, en l'équipement des Cases de santé concernées par l'expérimentation et la mise à disposition du matériel de travail des agents $\mathrm{SBC}$, en la tenue de réunions de coordination et de planification des activités, et au lancement officiel des activités SBC.

La phase de fonctionnement ou de mise en œuvre des interventions SBC a porté sur les 12 mois qui ont suivi cette phase précédente. Ces activités incluent certaines activités initiées depuis la phase préparatoire, comme les réunions de coordination et de planification des activités ; les activités des ASBC (les causeries éducatives, les visites à domicile, les ventes de produits $\mathrm{SBC}$, les références et la production de statistiques de services); et les activités de supervision par les ICP.

\section{Sources de données}

Les données utilisées dans l'étude d'analyse des coûts proviennent de trois sources :

- les compte-rendus des réunions tenues dans le cadre du projet SBC;

- les documents comptables produits par l'agence de collaboration responsable du volet Intervention; et

- les rapports d'activités produits tous les deux mois par les ASC et RC.

\section{Nature des coûts}

Les coûts sont définis comme les mesures des coûts marginaux des activités de conception et de mise en œuvre de chacune des interventions, ainsi que les coûts additionnels des prestations de services. Ceux-ci englobent tous les coûts relatifs aux salaires, à la formation et à la supervision des agents de santé et autres personnels communautaires, l'administration, aux infrastructures, aux équipements et aux produits. Parmi les ressources engagées dans la mise en œuvre du projet SBC, on distingue :

- les ressources qui ont un coût financier connu entièrement pris en charge par MSH, responsable du volet Intervention : les perdiems de formation, la location de salle, les dépenses en équipements et matériels de travail des ASC et RC; et 
- les ressources qui sont des donations gracieusement offertes au projet par de tierces parties : salles de réunion, repas pour les participants, le temps consacré aux réunions du projet par des personnes-ressources du niveau central, du district ou de la communauté, le temps consacré aux activités du projet par les ASBC. Pour ces biens et services, les coûts, qui n'apparaissent pas au niveau des documents comptables du projet, sont estimés ${ }^{14}$.

Le projet SBC a aussi bénéficié de certaines opportunités trouvées sur le terrain d'expérimentation : l'engagement de l'équipe-cadre du district et des ICP qui officient au niveau des sites expérimentaux ; les réunions périodiques tenues au niveau des Postes de santé entre les ASBC et les ICP pour évaluer les activités SBC; la contribution des ICP à la qualité et à la remontée régulière des statistiques de services enregistrées par les ASBC. Ces services ne sont pas pris en compte dans l'analyse des coûts des modèles SBC avec ASC et avec RC expérimentés.

\section{Rentabilité}

Elle représente le coût marginal du recrutement de chaque nouvelle personne utilisant les services SBC. C'est le prix unitaire par cliente utilisant l'un ou l'autre des services de santé de la reproduction offerts : une méthode de contraception moderne, un traitement IST, un traitement de cas de paludisme anténatal. Le coût marginal du projet SBC, tel qu'expérimenté avec les 60 ASBC pour une durée de 12 mois, inclut les coûts des activités préparatoires et les coûts de mise en œuvre de chaque modèle communautaire expérimenté. Ces coûts peuvent être reportés à différentes mesures de performance réalisées par le projet durant la même période.

\section{Les indicateurs de performance}

La performance de chaque modèle peut être appréciée à travers :

- des indicateurs de résultats immédiats du projet SBC tirés des statistiques de service contenues dans les rapports d'activités: le nombre de clients servis en produits contraceptifs, en médicaments, ou référés par les ASBC ; le nombre d'activités IEC menées; et

- des indicateurs des effets des programmes obtenus à partir des enquêtes CAP pré et post intervention auprès des populations cibles : Connaissances des usagers en matière de services SR ; taux de prévalence. Les enquêtes CAP ont fourni des informations sur les niveaux de connaissances, attitudes et pratiques des populationscibles dans quatre composantes de la SR. Ils constituent autant d'indicateurs de performances qui peuvent être utilisés dans l'appréciation de l'efficacité et de la rentabilité des modèles SBC expérimentés.

Dans ce rapport on s'est limité aux indicateurs de résultats immédiats et au taux de prévalence contraceptive pour apprécier la performance de chaque modèle.

\footnotetext{
${ }^{14}$ Pour chaque salle de réunion et chaque repas un coût forfaitaire est retenu. A chaque fonctionnaire de l'Etat ayant pris part aux réunions, on affecte un salaire mensuel égal à la moyenne entre le salaire en début de carrière et le salaire en fin de carrière correspondant à sa catégorie socioprofessionnelle. Aux membres de la communauté non salariés et aux ASBC, supposés sous-utilisés dans le système économique, on affecte un revenu mensuel égal au SMIG (salaire minimum garanti) qui est de $209 \mathrm{Fcfa} /$ heure.
} 


\section{Résultats}

\section{Coûts des interventions communautaires}

Ils portent sur l'ensemble des activités communes aux modèles SBC expérimentés avec ASC et avec RC et sur les activités spécifiques à chacun de ces modèles.

- Coûts des activités communes aux modèles expérimentés

Les activités communes aux deux modèles SBC expérimentés ont été exécutées en aval de l'intervention et portent essentiellement sur les 5 principales activités de la phase préparatoire présentées dans le tableau $6 a$.

Tableau 6a : Coûts des activités communes aux modèles expérimentés

\begin{tabular}{lccc}
\hline Activités communes & Coût total & $\begin{array}{c}\text { Coût pris en charge } \\
\text { par le projet }\end{array}$ & $\begin{array}{c}\text { Contribution de } \\
\text { tiers }\end{array}$ \\
\hline 1. Concertations aux niveaux Central, District & 896891 & 0 & 896891 \\
$\quad$ et Communautaire & 11874813 & 11462325 & 412488 \\
2. Ateliers de formation du personnel du projet & 4500000 & 0 & 4500000 \\
3. Provision en Kits des ASBC & 279808 & 67450 & 212358 \\
4. Réunions de planification des activités & 2167500 & 1200000 & 967500 \\
5. Lancement officiel des activités SBC & $\mathbf{1 9 7 1 9 0 1 2}$ & $\mathbf{1 2 7 2 9 7 7 5}$ & $\mathbf{6 9 8 9 2 3 7}$ \\
\hline Total & & & \\
\hline \hline
\end{tabular}

- Coûts marginaux des modèles communautaires

Le coût marginal de chaque modèle communautaire est calculé à partir :

- du coût des activités communes aux modèles SBC expérimentés : ce coût est équitablement réparti entre les deux modèles SBC expérimentés ;

- du coût des activités spécifiques à chaque modèle expérimenté qui accompagnent leur mise en oeuvre.

Le tableau $6 \mathrm{~b}$ donne le coût marginal total de chaque modèle communautaire selon les activités préparatoires de l'intervention et les activités de la phase d'intervention. Les coûts relatifs au dépôt bimestriel des rapports d'activités SBC, aux causeries éducatives, aux visites à domicile et aux entretiens/counseling sont estimés à partir du temps consacré par les agents aux activités SBC. Ces coûts ne sont pas supportés par le projet.

En rapportant les données du tableau 6.b aux unités de référence ${ }^{15}$ de chaque modèle $\mathrm{SBC}$, on en déduit le coût marginal total moyen d'une case de santé du modèle SBC avec ASC à 973.361 francs CFA, et d'un binôme RC à 1.412.113 francs CFA. Ces statistiques montrent que le coût marginal total du modèle $\mathrm{SBC}$ avec $\mathrm{RC}$ est 1.45 fois plus élevé que celui du modèle SBC avec ASC. La disparité entre les deux modèles SBC est encore plus grande si l'on considère les coûts de fonctionnement. Sur la période de 12 mois d'intervention des modèles SBC, les coûts de fonctionnement d'une case de santé du modèle SBC avec ASC a été en moyenne de 157.727 francs CFA contre 292.322 francs CFA pour un binôme RC du modèle SBC avec RC. Ces statistiques montrent bien que le coût de fonctionnement du modèle SBC avec RC est 1.85 fois plus élevé que celui du modèle SBC avec ASC. Mais

\footnotetext{
${ }^{15}$ Pour le modèle SBC avec ASC, l'unité de référence est la case de santé fonctionnelle ; et pour le modèle SBC avec RC, l'unité de référence est constituée par le binôme RC
} 
l'importance de cet écart s'explique principalement par les coûts assez élevés du moyen de transport alloué aux RC.

Tableau 6b : Coûts marginaux des modèles expérimentés

\begin{tabular}{|c|c|c|}
\hline Type d'activité & Modèle ASC & Modèle RC \\
\hline Activités préparatoires de l'intervention & 9859506 & 9859506 \\
\hline \multicolumn{3}{|l|}{ Activités de mise en œuvre de l'intervention } \\
\hline - $\quad$ Equipement des Cases de santé & 2375000 & 0 \\
\hline - Achat des chevaux et charrettes & 0 & 6937350 \\
\hline - Dépôts bimestriels des rapports SBC & 90600 & 0 \\
\hline - Supervision des ASBC & 266825 & 266825 \\
\hline - Suivi vétérinaire des chevaux & 0 & 262500 \\
\hline - Entretien cheval et charrette & 0 & 1440000 \\
\hline - Causeries éducatives de groupe & 1171440 & 1982880 \\
\hline - Visites à domicile & 733590 & 413820 \\
\hline - Entretiens/counseling & 103455 & 18810 \\
\hline Ensemble & 14600416 & 21181691 \\
\hline
\end{tabular}

Ces données révèlent également l'importance relative des coûts des activités de la phase préparatoire qui représentent $84 \%$ et $80 \%$ de l'ensemble des coûts pour le modèle ASC et pour le modèle $\mathrm{RC}$ respectivement. La contribution des donations à la prise en charge du coût total de chacun de ces modèles est du même ordre d'importance : $38 \%$ pour le modèle ASC et $35 \%$ pour le modèle RC.

\section{Rentabilité des modèles communautaires}

La rentabilité de chaque modèle SBC a été évaluée par rapport au coût marginal total et par rapport au coût marginal supporté par le budget du projet. Les tableaux $7 \mathrm{a}$ et $7 \mathrm{~b}$ donnent pour le modèle ASC et pour le modèle RC, l'ensemble des statistiques synthétisant cette situation. La deuxième colonne de ces tableaux donne les indicateurs de performances de chacun des modèles communautaires au cours des 10 premiers mois de l'expérimentation. La troisième et la quatrième colonnes indiquent respectivement le coût marginal par client servi par rapport au coût global de conception et de mise en œuvre et par rapport au coût marginal supporté par le budget du projet sans tenir compte des contributions de tierces parties. 
Tableau 7a : Rentabilité du modèle communautaire avec ASC

\begin{tabular}{|c|c|c|c|}
\hline \multirow[b]{2}{*}{ Indicateurs de performance } & \multirow[b]{2}{*}{$\begin{array}{l}\text { Evolution de } \\
\text { l'indicateur }\end{array}$} & \multicolumn{2}{|c|}{ Coût marginal par client servi* } \\
\hline & & $\begin{array}{c}\text { Par rapport au coût } \\
\text { total du modèle } \\
(14600416 \mathrm{Fcfa}) \\
\end{array}$ & $\begin{array}{c}\text { Par rapport au coût du } \\
\text { modèle supporté par le } \\
\text { projet }(9006713 \mathrm{cfa})\end{array}$ \\
\hline $\begin{array}{l}\text { Nombre de clients servis en méthodes } \\
\text { contraceptives }^{16}\end{array}$ & 191 & 76442 & 47156 \\
\hline Nombre de clients servis en médicaments ${ }^{17}$ & 2553 & 5719 & 3528 \\
\hline $\begin{array}{l}\text { Nombre de clients référés vers un Poste ou } \\
\text { Centre de santé }\end{array}$ & 469 & 31131 & 19204 \\
\hline Nombre de clients servis par le modèle & 3213 & 4544 & 2803 \\
\hline \multicolumn{4}{|l|}{$*$ en francs CFA. } \\
\hline \multirow[b]{2}{*}{ Indicateurs de performance } & \multirow{2}{*}{$\begin{array}{l}\text { Evolution de } \\
\text { l'indicateur }\end{array}$} & \multicolumn{2}{|c|}{ Coût marginal par client servi* } \\
\hline & & $\begin{array}{c}\text { Par rapport au coût } \\
\text { total du modèle } \\
(21181691) \\
\end{array}$ & $\begin{array}{c}\text { Par rapport au coût du } \\
\text { modèle supporté par le } \\
\text { projet (13 831 563) }\end{array}$ \\
\hline $\begin{array}{l}\text { Nombre de Clients servis en méthode } \\
\text { contraceptive }\end{array}$ & 258 & 82100 & 53611 \\
\hline Nombre de Clients servis en médicaments ${ }^{20}$ & 3335 & 6351 & 4147 \\
\hline $\begin{array}{l}\text { Nombre de Clients référés vers un Poste ou } \\
\text { Centre de santé }\end{array}$ & 653 & 32438 & 21182 \\
\hline Nombre de clients servis par le modèle & 4246 & 4989 & 3258 \\
\hline
\end{tabular}

Les données de ces tableaux révèlent que, quel que soit l'indicateur de résultat considéré, le coût marginal par unité de client servi est plus élevé avec le modèle SBC avec RC qu'avec le modèle SBC avec ASC. La différence est faible avec les ventes de médicaments et les références. Mais elle est statistiquement significative quand il s'agit du nombre de clients servis en PF. L'analyse de la rentabilité des modèles SBC peut être aussi faite à partir du coût de recrutement d'une cliente PF pour chacun des 2 modèles SBC:

- Dans la zone d'expérimentation du modèle SBC avec ASC, la prévalence contraceptive est passée de $5.7 \%$ avant l'intervention à $7.8 \%$ après l'intervention, soit une augmentation de 2.1 points. En supposant que le coût de fonctionnement de ce modèle (2.365.910 francs CFA) a engendré cet accroissement de 2.1 points sur le taux de prévalence contraceptive de la zone d'étude, l'on peut conclure que pour obtenir un accroissement d'une unité du taux de prévalence contraceptive, il faudrait en moyenne un coût de fonctionnement de 1.126.624 francs CFA. Ce qui implique que, pour le recrutement d'une nouvelle cliente PF, il faudrait un coût annuel de fonctionnement de 204.841 francs CFA.

\footnotetext{
${ }^{16} 135$ en préservatifs, 46 clients en pilules et 10 clients en spermicide

17946 clients en chloroquine, 873 en Fer, 627 en Vitamine A et 107 en sachets de SRO

${ }^{18} 212$ pour consultation prénatale ou postnatale, 162 pour la survie de l'enfant (vaccination, pesées, traitement de maladies diarrhéiques, traitement du paludisme...), 59 pour la planification familiale, 8 pour les IST et 28 pour diverses autres raisons

19145 clients en spermicides, 105 clients en préservatifs et 8 clients en préservatifs

${ }^{20} 950$ en paracétamol, 916 en Vitamine A, 726 en chloroquine, 569 en Fer et 174 en sachet de SRO

${ }^{21}$ Références : 174 pour la maternité à moindre risque, 127 pour la survie de l'enfant 127, 28 pour la PF, 59 pour les IST et diverses autres raisons telles que la consultation, les soins curatifs, etc.
} 
- $\quad$ De même pour le modèle SBC avec RC le coût de fonctionnement de 4.384.835 francs CFA a engendré une augmentation de 0.9 points sur la prévalence contraceptive de la zone d'étude, car la prévalence est passée de $3.3 \%$ avant l'intervention à $4.2 \%$ après l'intervention. On en déduit que le coût marginal pour une unité d'augmentation du taux de la prévalence contraceptive est en moyenne égal à un coût moyen de fonctionnement de 4.989.150 francs CFA équivalent à un coût de fonctionnement annuel de 885.825 francs CFA pour le recrutement d'une nouvelle cliente PF.

Le rapport entre ces deux résultats montre que le coût marginal pour le recrutement d'une cliente PF du modèle SBC avec RC est 4.3 fois celui du modèle SBC avec ASC. Ainsi tous les indicateurs de rentabilité des tableaux ci-dessus mènent à la même conclusion que le modèle ASC est plus rentable que le modèle RC. Mais vu le niveau des indicateurs, la rentabilité de chacun de ces modèles est jugée trop faible.

\section{Dissémination et Utilisation des résultats}

Les données collectées dans le cadre de cette expérimentation ont fait l'objet d'utilisation sous diverses formes.

Les données de l'Analyse Situationnelle de base : Elles ont servi dans la détermination des besoins des structures de santé concernées par l'expérimentation en équipement et en formation.

Les données des enquêtes CAP de base : Elles ont servi dans l'identification et la conception des thèmes du curriculum de formation des ASBC.

Dissémination des résultats: Une journée de dissémination des résultats de l'expérimentation SBC a été organisée au niveau district. Cette journée a permis un premier et large partage, entre les acteurs aux niveaux local et central, des résultats obtenus dans le cadre du projet d'expérimentation SBC de Kébémer. Ce séminaire de dissémination était présidé par le Préfet de Kébémer, la plus haute autorité administrative du département. Près d'une centaine de participants y ont pris part. Parmi ces participants, on comptait le Maire de la commune, les 10 Présidents de Communautés Rurales du département de Kébémer, 12 agents SBC du projet (6 ASC et 6RC), les $11 \mathrm{ICP}$ du district, tout le personnel du Centre de santé de Kébémer, le Médecin Chef de la région de Louga, le chef de la DSR et le responsable technique du projet, et les représentants des agences de collaboration et ONGs travaillant dans le district. Dix médias locaux ont couvert l'événement et les discours d'ouverture du Préfet ainsi que celui du Maire ont été retransmis en direct sur les ondes de la principale radio locale qui couvre tout le département de Kébémer.

Six journées de dissémination des résultats au niveau communautaire ont également été organisées pour présenter et discuter les résultats spécifiques à chaque communauté rurale. Les modèles d'offre de services à base communautaire de services de SR expérimentés ont également été exposés devant le Ministre de la Santé et de la Prévention lors d'une journée focale au niveau District et dans le cadre d'un séminaire de formation des médecins en santé publique. 
Perspectives d'utilisation des résultats : Suite à l'atelier de dissémination des résultats au niveau du District, le comité technique de suivi du projet s'est réuni deux fois ${ }^{22}$ pour faire des réflexions et propositions concrètes sur les suites à donner à l'expérimentation des deux modèles SBC. Au cours de ces deux réunions techniques, la DSR a noté l'importance des acquis au terme du projet:

- le savoir-faire acquis par la DSR dans la pratique d'intervention en SBC est un résultat à magnifier ;

- les résultats de l'expérimentation ont été discutés au niveau de la première réunion de coordination du District Sanitaire de Kébémer qui a suivi la fin du projet. Ainsi l'approche SBC a été jugée intéressante par les responsables qui ont souhaité qu'elle soit étendue à l'ensemble du District ;

- les communautés desservies par les modèles communautaires expérimentés souhaitent vivement la continuation des activités SBC;

- l'expérience de Kébémer suscite déjà l'intérêt de diverses personnes-ressources qui envisagent de faire une visite de terrain ;

- la méthodologie du projet a déjà été répliquée par des ONGs et agences de collaboration dans d'autres régions du Sénégal: AFRICARE et ASBEF à Kaolack ; ACDEV à Fatick; et FANKATA à Kolda.

- les fiches de collecte et de synthèse des données produites par le projet ont été jugées très utiles par les ICP. Dores et déjà, la multiplication de ces outils de collecte de données en quantité suffisante a été inscrite dans les plans d'opérations de 2004 de la DSR. Elle sera financée par des fonds du projet bilatéral de l'USAID.

En termes de pérennisation de l'approche, le comité technique a planifié les activités suivantes :

- La restitution des résultats de l'expérience SBC au niveau Poste de santé et au niveau de la communauté. Cette restitution se fera sous forme de briefings avec tous les ICP et les collectivités locales. Le comité technique a recommandé au District sanitaire de Kébémer de réunir dans l'immédiat les ICP concernés par l'expérience SBC pour discuter avec eux des perspectives de l'après projet; et de les informer des activités de restitution au niveau Poste. Population Council a été sollicité pour la prise en charge des activités de restitution au niveau communautaire.

- La prise en compte de l'approche SBC dans les plans d'opérations des districts qui est le processus de planification des activités de santé de la reproduction au niveau communautaire.

- La validation et la standardisation du curriculum de formation des ASBC ainsi que des instruments de collecte et de synthèse des données: Cette activité sera inscrite dans les plans d'opérations de 2004 de la DSR et financée par le projet bilatéral de l'USAID.

- L'organisation par la DSR d'un atelier national d'une journée pour partager les résultats de l'expérience.

- La sélection du District sanitaire de Kébémer comme district de référence en matière de $S B C$ : Dans le cadre de la pérennisation des activités SBC, le District sanitaire de Kébémer doit sélectionner au moins deux communautés rurales comme zone pilote et y apporter tout l'appui technique nécessaire en matière de SBC dans les plus brefs délais. Cette zone sera retenue comme zone de visite de l'expérience. La DSR donnera toute l'assistance nécessaire pour la réalisation de cette activité.

${ }^{22}$ Le 27 septembre 2003 au bureau du Population Council et le 31 octobre 2003 à la DSR 
- Le développement d'un protocole d'expansion des activités $S B C$ dans les districts USAID et la recherche de financement auprès des bailleurs de fonds pour l'exécution de ce projet. Le comité technique a sollicité l'assistance de Population Council à la DSR pour la réalisation de cette tâche. 


\section{CONCLUSIONS ET RECOMMANDATIONS}

En résumé, aucun des modèles n'a réellement réussi à augmenter de façon significative les connaissances attitudes et pratiques de planification familiale par rapport à la zone de comparaison. Cependant, la zone de comparaison était dans un site plus urbanisé et développé que les deux sites DBC sis dans des zones rurales largement inaccessibles, et par conséquent avait des niveaux de connaissances attitudes et pratiques beaucoup plus élevés.

Malgré l'existence de besoins non satisfaits en PF $(32,3 \%)^{23}$, ces modèles d'intervention SBC n'ont pas réussi à augmenter de manière significative la pratique contraceptive dans les zones d'expérimentation respectives des modèles SBC. Plusieurs facteurs peuvent être avancés pour expliquer cette situation : la durée de la période d'expérimentation de 12 mois jugée trop courte, l'absence d'une stratégie IEC globale adaptée au contexte local et ciblant la PF pour renforcer les activités IEC des modèles SBC et davantage susciter la demande, et les conditions d'application des modèles. Ce dernier aspect est d'importance car il dénote un hiatus entre les prévisions initiales contenues dans le protocole de recherche et les activités effectivement réalisées. Cet hiatus porte principalement sur:

- la prise en charge de la supervision en particulier au niveau Poste de santé ;

- l'organisation de réunions trimestrielles au niveau des Postes de santé ;

- le nombre de sessions de recyclage des ASBC;

- l'expérimentation de l'offre de pilules en première prescription par les ASBC ;

- le complément d'équipement pour le renforcement de la capacité fonctionnelle des Postes et du Centre de santé ;

- le recrutement du coordonnateur SBC au niveau district et la mise à sa disposition de moyens adéquats.

Il ressort de cet écart constaté que les modèles SBC expérimentés n'ont pas été appliqués tels que décrits dans le protocole de recherche.

La faisabilité technique de ces deux modèles SBC est moins évidente si l'on en juge à travers les performances des ASBC, leurs opinions sur la formation reçue et le système de supervision, et les problèmes de mise en œuvre mentionnés plus haut.

Malgré ces résultats quantitatifs quelque peu mitigés, les populations des zones du projet, le personnel de santé du district ainsi que les hauts responsables de la santé au niveau central ont jugé acceptables les deux modèles SBC expérimentés. Pour les populations cibles, ces modèles SBC ont davantage amélioré l'accès aux services de SR. Parmi les apports positifs du projet, l'on peut noter la disponibilité de nouveaux instruments de collecte de données au niveau communautaire (fiches pictographiques et autres fiches de synthèse) qui sont d'une importance capitale pour les ICP, l'élaboration, pour la première fois au Sénégal, d'un curriculum de formation pour les agents SBC, la documentation de l'approche telle qu'elle a été expérimentée, et l'expérience acquise par le personnel de la DSR sur la méthodologie de l'approche SBC. Ils constituent autant de résultats que la DSR doit valider, standardiser et promouvoir l'utilisation. La réalisation de cette tâche passera nécessairement par le développement d'un plan d'utilisation de ces résultats par la DSR.

Il est aussi important de souligner que l'analyse des coûts du projet SBC constitue une première du genre au Sénégal. Elle a permis de rassembler des informations de base sur les

\footnotetext{
${ }^{23}$ Enquête Démographique et de Santé au Sénégal (EDS III), Ministère de l'Economie, des Finances et du Plan, et Macro International Inc, décembre 1997, 144 pages + annexes.
} 
coûts qui, jusqu'ici, n'existaient pas. Ces informations constitueront une référence de base utile aux responsables de programmes dans la perspective d'une réplication ou d'une expansion de ces modèles SBC au niveau national.

Pour la réplication des modèles SBC expérimentés, il est à savoir que le modèle ASC est moins coûteux que le modèle RC. Les résultats de l'analyse des coûts révèlent que le coût marginal moyen de la phase préparatoire est 1.37 fois plus élevé dans modèle $\mathrm{RC}$ que dans le modèle ASC ; et que les coûts de fonctionnement du modèle RC sont 1.85 fois ceux du modèle ASC. Le coût de fonctionnement annuel d'une Case de santé du modèle ASC est en moyenne de 157.727 francs CFA contre 292.322 francs CFA pour un binôme RC. La part des donations faites par des tiers dans le coût total des activités de la phase préparatoire des interventions s'élève à environ $35 \%$. Tous ces résultats sont importants pour les responsables de programme pour une bonne planification et mise en œuvre des activités des phases de préparation et de fonctionnement des SBC. Pour le décideur, les résultats de l'étude montrent également que le modèle ASC présente une meilleure rentabilité. Le coût de recrutement d'une nouvelle cliente PF calculé à partir du coût de fonctionnement de chaque modèle sur une période de 12 mois montre que ce coût dans le modèle RC (885.825 francs CFA) est 4.3 fois celui du modèle ASC (204.841 francs CFA).

Parmi les enseignements à tirer au terme de cette expérience du District de Kébémer l'on retiendra que :

- Le modèle RC considéré comme une alternative pour remplacer les Cases de Santé non fonctionnelles, s'est avéré moins efficace et plus coûteux que le modèle ASC qui renforce la capacité des ASC travaillant dans les Cases de Santé fonctionnelles. En conséquence, là où il existe des Cases de santé fonctionnelles le renforcement de ces structures apparaît comme un moyen approprié pour desservir les populations rurales. Dans les zones où il n'y en a pas, il semble plus indiqué et approprié pour la DSR de rendre ces Cases de Santé fonctionnelles et ensuite chercher à renforcer la capacité de fonctionnement de leur personnel : c'est à dire les ASCs.

- Dans le processus de mise en œuvre d'un projet SBC, la phase préparatoire, en particulier l'étape des concertations communautaires, s'avère d'une extrême importance pour la participation et l'implication de la communauté. Des instances de coordination administrative telles que le Comité Départemental de Développement (CDD) et le Comité Local de Développement (CLD) peuvent être des canaux efficaces d'information des partenaires locaux ;

- Parmi les critères de choix d'un ASBC, celui lié à l'instruction ou à l'alphabétisation est d'une grande importance. Un ASBC doit nécessairement être instruit ou alphabétisé pour répondre aux exigences de la stratégie $\mathrm{SBC}$;

- Le système d'information dans le cadre d'une stratégie SBC doit s'appuyer sur des outils et supports de collecte adaptés aux ASBC. La fiche pictographique se révèle adaptée à des ASBC analphabètes ou d'un très faible niveau d'instruction ;

- Dans le cadre de la mise en œuvre d'une stratégie SBC, la communauté ainsi que les ASBC eux-mêmes, doivent être étroitement associés au choix du moyen de transport; et chaque ASBC doit avoir son autonomie en termes de moyens de déplacements ;

- Le choix d'une stratégie mobile basée sur la traction chevaline nécessite que soit mis en place un système adéquat de suivi vétérinaire ;

- La diversification des services et des produits offerts (PF et autres médicaments essentiels) permet une meilleure acceptabilité des ASBC au sein des communautés ; 
- Dans le cadre de la mise en œuvre d'un projet SBC, l'engagement de l'ICP et la supervision régulière des activités sont des éléments essentiels ;

- Un système d'approvisionnement en produits assuré par le superviseur direct (ICP) permet d'éviter les ruptures de stocks ;

- Le système de motivation fondé sur la seule marge bénéficiaire réalisée sur les ventes de produits SBC est insuffisant. Il est nécessaire d'explorer d'autres sources de motivation ;

- L'installation officielle de l'ASBC au sein de sa communauté constitue un élément important de reconnaissance sociale et de valorisation du travail de l'ASBC.

Enfin, pour maximiser la performance de 1 'ASBC, accroître ses revenus et assurer la pérennisation des programmes $\mathrm{SBC}$, il sera nécessaire de mener une étude RO sur le meilleur mode d'association ou d'intégration des produits $\mathrm{PF}$ et de santé dans ces programmes SBC dans le contexte social sénégalais. 


\section{ANNEXES}

Tableau 2a : Caractéristiques socio-démographiques des femmes selon la zone d'étude

Echantillon des Femmes

\begin{tabular}{|c|c|c|c|c|c|c|}
\hline & \multicolumn{2}{|c|}{ Zone RC } & \multicolumn{2}{|c|}{ Zone ASC } & \multicolumn{2}{|c|}{ Zone de comparaison } \\
\hline & $\begin{array}{c}\text { Pré } \\
(n=550)\end{array}$ & $\begin{array}{c}\text { Post } \\
(n=560)\end{array}$ & $\begin{array}{c}\text { Pré } \\
(n=550)\end{array}$ & $\begin{array}{c}\text { Post } \\
(n=553)\end{array}$ & $\begin{array}{c}\text { Pré } \\
(n=550)\end{array}$ & $\begin{array}{c}\text { Post } \\
(n=561)\end{array}$ \\
\hline \multicolumn{7}{|l|}{ Age } \\
\hline - moyen & 29,3 & 29,3 & 28,9 & $28,5^{*}$ & 28,2 & 28 \\
\hline - médian & 28 & 28 & 28 & 27 & 27 & 27 \\
\hline \multicolumn{7}{|l|}{ Instruction (\%) } \\
\hline \multicolumn{7}{|c|}{$\begin{array}{l}\text { - pas d'instruction / } \\
\text { alphabétisée / école }\end{array}$} \\
\hline coranique & 93,7 & $96,3^{*}$ & 95,6 & 95,3 & 44 & 43,3 \\
\hline - primaire & 5,5 & 3,2 & 4,2 & 4,7 & 37 & 37,1 \\
\hline - secondaire & 0,8 & 0,5 & 0,2 & --- & 18 & 18,7 \\
\hline - supérieur & --- & --- & --- & --- & 1 & 0,9 \\
\hline \multicolumn{7}{|c|}{ Situation matrimoniale $(\%)$} \\
\hline - mariée & 94,2 & 94,3 & 91,8 & 92,2 & 69,3 & 69 \\
\hline - célibataire & 5,5 & 5,2 & 7,6 & 6,9 & 28,5 & 28,3 \\
\hline - div/sép/Veuve & 0,3 & 0,5 & 0,6 & 0,9 & 2,2 & 2,7 \\
\hline \multicolumn{7}{|c|}{ Nombre de grossesses } \\
\hline - moyen & 4 & $3,7 *$ & 3,9 & $3,6^{*}$ & 2,8 & 2,8 \\
\hline - médian & 4 & 3 & 3 & 3 & 2 & 2 \\
\hline \multicolumn{7}{|c|}{ Nombre de naissances vivantes } \\
\hline - moyen & 3,5 & $3,1 *$ & 3,4 & $3,2 *$ & 2,4 & 2,4 \\
\hline - médian & 3 & 3 & 3 & 3 & 2 & 2 \\
\hline \multicolumn{7}{|c|}{ Age (en mois) du dernier enfant } \\
\hline - moyen & 33,1 & $38,6^{*}$ & 27,9 & $34,2 *$ & 43,1 & $46,9 *$ \\
\hline - médian & 23 & 24 & 18 & 24 & 27 & 30 \\
\hline
\end{tabular}

Source : Enquêtes CAP Femmes Pré-intervention (2001) et Post-intervention (2003) ; * significatif à 5 \%. 
Tableau 2b : Caractéristiques socio-démographiques des hommes selon la zone d'étude

Echantillon des Hommes

\begin{tabular}{|c|c|c|c|c|c|c|}
\hline & \multicolumn{2}{|c|}{ Zone RC } & \multicolumn{2}{|c|}{ Zone ASC } & \multicolumn{2}{|c|}{ Zone de comparaison } \\
\hline & $\begin{array}{r}\text { Pré } \\
(n=500)\end{array}$ & $\begin{array}{c}\text { Post } \\
(n=499)\end{array}$ & $\begin{array}{r}\text { Pré } \\
(n=500)\end{array}$ & $\underset{(n=456)}{\text { Post }}$ & $\begin{array}{c}\text { Pré } \\
(\mathbf{n}=500)\end{array}$ & $\begin{array}{c}\text { Post } \\
(n=507)\end{array}$ \\
\hline \multicolumn{7}{|l|}{ Age } \\
\hline - moyen & 35,9 & $40,9 *$ & 33 & $37,1 *$ & 32 & $33,2 *$ \\
\hline - médian & 33 & 40 & 28 & 36 & 29 & 30 \\
\hline \multicolumn{7}{|l|}{ Instruction (\%) } \\
\hline \multicolumn{7}{|c|}{$\begin{array}{l}\text { - pas d'instruction / } \\
\text { alphabétisé / école }\end{array}$} \\
\hline coranique & 92,4 & 91 & 94 & 95,8 & 37,4 & 39,6 \\
\hline - primaire & 5,4 & 6,4 & 5,2 & 2,4 & 25,2 & 24,3 \\
\hline - secondaire & 2 & 2 & 0,8 & 1,1 & 27,4 & 26,4 \\
\hline - supérieur & 0,2 & 0,6 & --- & 0,7 & 10 & 9,7 \\
\hline \multicolumn{7}{|c|}{ Situation matrimoniale $(\%)$} \\
\hline - marié & 69,6 & $78,2 *$ & 60,2 & $73,2 *$ & 45 & 45,8 \\
\hline - célibataire & 30,2 & $20,6^{*}$ & 38,4 & $25,4 *$ & 52 & 52,1 \\
\hline - div/sép/Veuf & 0,2 & 1,2 & 1,4 & 1,4 & 3 & 2,1 \\
\hline
\end{tabular}

Source : CAP Hommes Pré-intervention (2001) et Post-intervention (2003); * significatif à $5 \%$. 
Tableau 8 : Indice de faisabilité des modèles communautaires selon les RC et les ASC

\begin{tabular}{|c|c|c|c|}
\hline Procédures & $\begin{array}{c}\text { RC } \\
(n=27)\end{array}$ & $\begin{array}{c}\text { ASC } \\
(n=25)\end{array}$ & $\begin{array}{l}\text { Test de } \\
\text { Fischer }\end{array}$ \\
\hline \multicolumn{4}{|l|}{ Mise en œuvre des modèles } \\
\hline 1. Ont réalisé des activités IEC & 0.96 & 1 & .519 \\
\hline 2. Ont vendu des produits SBC & 1 & 0.96 & .481 \\
\hline 3. Ont référé des clients & 0.85 & 1 & .065 \\
\hline 4. Ont utilisé les affiches & 1 & 1 & -- \\
\hline 5. Ont utilisé le mannequin & 0.74 & 0.80 & .431 \\
\hline 6. Ont utilisé les cartes IST & 0.96 & 0.96 & .735 \\
\hline 7. Ont utilisé l'échantillon de produits contraceptifs & 0.96 & 1 & .519 \\
\hline 8. Ont utilisé les dépliants & 0.93 & 1 & .265 \\
\hline 9. N'ont jamais connu de rupture de stock & 0.48 & 0.44 & .492 \\
\hline \multirow[t]{2}{*}{$\begin{array}{l}\text { 10. N'ont pas eu des problèmes relatifs au remplissage des } \\
\text { fiches pictographiques }\end{array}$} & 0.56 & 0.36 & .128 \\
\hline & & & Anova \\
\hline Valeur moyenne de l'indice & 8.4 & 8.5 & .780 \\
\hline \multicolumn{4}{|l|}{ Ont bien apprécié la formation reçue } \\
\hline 1. La durée de la formation était suffisante & 0.58 & 0.63 & .483 \\
\hline 2. Le contenu de la formation était suffisant & 0.77 & 0.71 & .429 \\
\hline 3. Le nombre de sessions de recyclage était suffisant & 0.46 & 0.38 & .368 \\
\hline \multirow[t]{2}{*}{ 4. En définitive la formation reçue était appropriée } & 0.59 & 0.60 & .590 \\
\hline & & & Anova \\
\hline Valeur moyenne de l'indice & 2.3 & 2.2 & .827 \\
\hline
\end{tabular}

Source : Interview ASBC 
Tableau 9 : Indice synthétique du niveau de connaissance en PF

\begin{tabular}{|c|c|c|c|c|c|c|}
\hline \multirow[b]{2}{*}{ Indicateurs } & \multicolumn{3}{|c|}{ Pré-intervention } & \multicolumn{3}{|c|}{ Post-intervention } \\
\hline & $\begin{array}{c}\mathrm{RC} \\
(n=550)\end{array}$ & $\begin{array}{c}\mathrm{ASC} \\
(n=550)\end{array}$ & $\begin{array}{l}\text { COMP } \\
(n=550) \\
\end{array}$ & $\begin{array}{c}\mathrm{RC} \\
(n=560)\end{array}$ & $\begin{array}{c}\mathrm{ASC} \\
(n=553) \\
\end{array}$ & $\begin{array}{l}\text { COMP } \\
(n=561)\end{array}$ \\
\hline $\begin{array}{l}1 \text { - Ont entendu parler de moyen de PF } \\
2 \text { - Citent spontanément au moins une méthode du } \\
\text { Projet } \\
3 \text { - Citent au moins un avantage de la pilule } \\
4 \text { - Citent au moins un avantage du préservatif } \\
5 \text { - Citent au moins un avantage du spermicide } \\
6 \text { - Citent la santé de la mère et de l'enfant comme } \\
\text { un avantage de l'espacement des naissances } \\
7 \text { - Citent le bien-être familial comme un avantage } \\
\text { de l'espacement des naissances }\end{array}$ & $\begin{array}{c}0.80 \\
0.50 \\
0.49 \\
0.49 \\
0.5 \\
0.71 \\
0.31\end{array}$ & $\begin{array}{c}0.67 \\
0.53 \\
0.42 \\
0.45 \\
0.8 \\
0.60\end{array}$ & $\begin{array}{c}0.93 \\
0.70 \\
0.44 \\
0.68 \\
0.9 \\
0.86\end{array}$ & $\begin{array}{l}0.93^{*} \\
0.76^{*} \\
0.69^{*} \\
0.70^{*} \\
0.30^{*} \\
0.90^{*} \\
0.36\end{array}$ & $\begin{array}{l}0.92^{*} \\
0.81^{*} \\
0.75^{*} \\
0.75^{*} \\
0.35^{*} \\
0.85^{*} \\
0.36\end{array}$ & $\begin{array}{c}0.96 \\
0.87^{*} \\
0.85^{*} \\
0.91^{*} \\
0.22^{*} \\
0.88 \\
0.39\end{array}$ \\
\hline $\begin{array}{l}\text { Indice calculé sur les } 7 \text { items } \\
\text { Variance } \\
\text { Indice sur une échelle de } 10 \text { points possibles }\end{array}$ & $\begin{array}{c}3.34 \\
4.294 \\
4.77\end{array}$ & $\begin{array}{c}3.04 \\
6.060 \\
4.34\end{array}$ & $\begin{array}{c}4.14 \\
3 \\
5.91\end{array}$ & $\begin{array}{c}4.64 \\
2.710 \\
6.63 *\end{array}$ & $\begin{array}{l}4.78 \\
2.836 \\
6.83 *\end{array}$ & $\begin{array}{c}5.08 \\
1.584 \\
7.26 *\end{array}$ \\
\hline
\end{tabular}

Sources : CAP femmes pré-intervention (2001) et post-intervention (2003).

$* \mathrm{p}<.05$ 
Tableau 10 : Indice synthétique du niveau de connaissance en IST/VIH/SIDA

\begin{tabular}{|c|c|c|c|c|c|c|}
\hline \multirow[b]{2}{*}{ Indicateurs } & \multicolumn{3}{|c|}{ Pré-intervention } & \multicolumn{3}{|c|}{ Post-intervention } \\
\hline & $\begin{array}{c}\mathrm{RC} \\
(n=550)\end{array}$ & $\begin{array}{c}\text { ASC } \\
(n=550)\end{array}$ & $\begin{array}{l}\text { COMP } \\
(n=550)\end{array}$ & $\begin{array}{c}\mathrm{RC} \\
(n=560)\end{array}$ & $\begin{array}{c}\mathrm{ASC} \\
(n=553)\end{array}$ & $\begin{array}{l}\text { COMP } \\
(n=561)\end{array}$ \\
\hline $\begin{array}{l}1 \text { - Ont entendu parler du VIH/SIDA } \\
2 \text { - Ont entendu parler d'IST autres que le SIDA } \\
3 \text { - Ont cité le préservatif comme moyen de } \\
\text { prévention du VIH/SIDA } \\
4 \text { - Ont cité la fidélité à un partenaire unique } \\
\text { comme moyen de prévention du } \\
\text { VIH/SIDA } \\
5 \text { - Ont cité au moins le préservatif et la fidélité à } \\
\text { un partenaire unique comme moyen } \\
\text { de prévention du VIH/SIDA }\end{array}$ & $\begin{array}{l}0.85 \\
0.46 \\
0.32\end{array}$ & $\begin{array}{l}0.87 \\
0.42 \\
0.25\end{array}$ & $\begin{array}{l}0.98 \\
0.52 \\
0.37\end{array}$ & $\begin{array}{c}0.98^{*} \\
0.60^{*} \\
0.39 \\
0.62\end{array}$ & $\begin{array}{l}0.98^{*} \\
0.62^{*} \\
0.40^{*}\end{array}$ & $\begin{array}{c}1^{*} \\
0.69^{*} \\
0.55^{*} \\
0.71^{*}\end{array}$ \\
\hline $\begin{array}{l}\text { Indice calculé sur les } 5 \text { items listés } \\
\text { Variance } \\
\text { Indice calculé sur une échelle de } 10 \text { points possibles }\end{array}$ & $\begin{array}{c}2.41 \\
2.118 \\
4.82\end{array}$ & $\begin{array}{c}2.37 \\
1.724 \\
4.74\end{array}$ & $\begin{array}{c}2.7 \\
1.485 \\
5.4\end{array}$ & $\begin{array}{l}2.84 \\
1.770 \\
5.68 *\end{array}$ & $\begin{array}{l}2.96 \\
1.770 \\
5.92 *\end{array}$ & $\begin{array}{l}3.31 \\
1.629 \\
6.62 *\end{array}$ \\
\hline
\end{tabular}

Sources : CAP femmes pré-intervention (2001) et post-intervention (2003).

$* \mathrm{p}<.05$ 
Tableau 11 : Indice synthétique du niveau de connaissance en maternité à moindre risque

\begin{tabular}{|c|c|c|c|c|c|c|}
\hline \multirow[b]{2}{*}{ Indicateurs } & \multicolumn{3}{|c|}{ Pré-intervention } & \multicolumn{3}{|c|}{ Post-intervention } \\
\hline & $\begin{array}{c}\mathrm{RC} \\
(n=550) \\
\end{array}$ & $\begin{array}{c}\mathrm{ASC} \\
(n=550) \\
\end{array}$ & $\begin{array}{l}\text { COMP } \\
(n=550) \\
\end{array}$ & $\begin{array}{c}\mathrm{RC} \\
(n=560) \\
\end{array}$ & $\begin{array}{c}\mathrm{ASC} \\
(n=553) \\
\end{array}$ & $\begin{array}{l}\text { COMP } \\
(n=561) \\
\end{array}$ \\
\hline \multirow{8}{*}{$\begin{array}{l}1 \text { - Savent que la } 1^{\text {ère }} \mathrm{CPN} \text { doit avoir lieu durant le premier trimestre de la grossesse } \\
2 \text { - Savent qu'une femme enceinte doit faire au moins } 3 \text { CPN durant sa grossesse } \\
3 \text { - Mentionnent qu'une femme enceinte doit se faire consulter pour savoir si elle est en bonne santé } \\
4 \text { - Mentionnent qu'une femme enceinte doit se faire consulter pour savoir si son enfant se porte bien } \\
5 \text { - Mentionnent qu'une femme enceinte doit se faire consulter pour prévenir certaines maladies } \\
6 \text { - Mentionnent qu'une femme enceinte doit se faire consulter pour suivre l'évolution de la grossesse } \\
7 \text { - Citent la chloroquine/quinine comme médicament que la femme enceinte doit systématiquement } \\
\text { prendre } \\
8 \text { - Citent le fer/folate comme médicament que la femme enceinte doit systématiquement prendre }\end{array}$} & 0.85 & 0.87 & 0.92 & 0.90 & 0.85 & 0.87 \\
\hline & 0.88 & 0.90 & 0.88 & 0.86 & 0.89 & 0.80 \\
\hline & 0.82 & 0.79 & 0.90 & 0.84 & $0.88^{*}$ & 0.90 \\
\hline & 0.69 & 0.72 & 0.83 & $0.78^{*}$ & 0.73 & 0.83 \\
\hline & 0.32 & 0.30 & 0.18 & 0.31 & 0.26 & $0.33^{*}$ \\
\hline & 0.24 & 0.29 & 0.25 & 0.18 & 0.24 & $0.47^{*}$ \\
\hline & 0.77 & 0.85 & 0.86 & 0.78 & 0.87 & 0.82 \\
\hline & 0.75 & 0.83 & 0.82 & 0.75 & 0.86 & 0.77 \\
\hline Indice calculé sur les 8 items & 5.32 & 5.55 & 5.65 & 5.40 & 5.58 & 5.78 \\
\hline Variance & 2.057 & 1.534 & 1.507 & 2.011 & 1.809 & 2.782 \\
\hline Indice calculé sur une échelle de 10 points possibles & 6.65 & 6.94 & 7.06 & $6.75 *$ & 6.98 & 7.23 \\
\hline
\end{tabular}

Sources : CAP femmes pré-intervention (2001) et post-intervention (2003).

$* \mathrm{p}<.05$ 
Tableau 12 : Indice synthétique du niveau de connaissance en Survie de l'enfant

\begin{tabular}{|c|c|c|c|c|c|c|}
\hline \multirow[b]{2}{*}{ Indicateurs } & \multicolumn{3}{|c|}{ Pré-intervention } & \multicolumn{3}{|c|}{ Post-intervention } \\
\hline & $\begin{array}{c}\mathrm{RC} \\
(n=403) \\
\end{array}$ & $\begin{array}{c}\mathrm{ASC} \\
(n=414) \\
\end{array}$ & $\begin{array}{l}\text { COMP } \\
(n=268) \\
\end{array}$ & $\begin{array}{c}\mathrm{RC} \\
(n=373) \\
\end{array}$ & $\begin{array}{c}\mathrm{ASC} \\
(n=383) \\
\end{array}$ & $\begin{array}{l}\text { COMP } \\
(n=259) \\
\end{array}$ \\
\hline $\begin{array}{l}\text { D - Survie de l'enfant } \\
1 \text { - Savent qu'un enfant doit prendre des vaccins pour sa survie } \\
2 \text { - Savent qu'il y a des maladies que l'on peut éviter avec les vaccins } \\
3 \text { - Connaissent le sachet de SRO } \\
4 \text { - Savent comment reconstituer la SRO } \\
5 \text { - Citent la solution domestique (eau + sel + sucre) comme traitement contre la diarrhée } \\
6 \text { - Savent qu'il faut administrer la solution à l'enfant à sa demande } \\
7 \text { - Citent la fièvre comme un signe du paludisme chez l'enfant } \\
8 \text { - Citent la chloroquine comme un moyen de prévention du paludisme chez l'enfant }\end{array}$ & $\begin{array}{l}0.98 \\
0.95 \\
0.52 \\
0.46 \\
0.67 \\
0.41 \\
0.89 \\
0.62\end{array}$ & $\begin{array}{l}0.99 \\
0.99 \\
0.59 \\
0.55 \\
0.80 \\
0.48 \\
0.89 \\
0.74\end{array}$ & $\begin{array}{c}1 \\
0.94 \\
0.80 \\
0.76 \\
0.79 \\
0.73 \\
1 \\
0.53\end{array}$ & $\begin{array}{c}1 \\
0.99 \\
0.73^{*} \\
0.62^{*} \\
0.67 \\
0.56^{*} \\
0.97^{*} \\
0.75^{*}\end{array}$ & $\begin{array}{c}1 \\
0.99 \\
0.77^{*} \\
0.70^{*} \\
0.75 \\
0.66^{*} \\
0.96^{*} \\
0.61^{*}\end{array}$ & $\begin{array}{c}1 \\
1 \\
0.91^{*} \\
0.87^{*} \\
0.80 \\
0.85^{*} \\
1 \\
0.82^{*}\end{array}$ \\
\hline $\begin{array}{l}\text { Indice calculé sur les } 8 \text { items } \\
\text { Variance } \\
\text { Indice calculé sur une échelle de } 10 \text { points possibles }\end{array}$ & $\begin{array}{c}5.49 \\
3.554 \\
6.86\end{array}$ & $\begin{array}{c}6.02 \\
2.864 \\
7.53\end{array}$ & $\begin{array}{c}6.53 \\
2.317 \\
8.16\end{array}$ & $\begin{array}{c}6.29 \\
2.598 \\
7.86 *\end{array}$ & $\begin{array}{c}6.42 \\
2.308 \\
\mathbf{8 . 0 3}\end{array}$ & $\begin{array}{l}7.25 \\
1.675 \\
9.06 *\end{array}$ \\
\hline
\end{tabular}

Sources : CAP femmes pré-intervention (2001) et post-intervention (2003).

$* \mathrm{p}<.05$ 
Tableau 13 : Indice synthétique des attitudes vis-à-vis de la PF

\begin{tabular}{|c|c|c|c|c|c|c|}
\hline \multirow[b]{2}{*}{ Indicateurs } & \multicolumn{3}{|c|}{ Pré-intervention } & \multicolumn{3}{|c|}{ Post-intervention } \\
\hline & $\begin{array}{c}\mathrm{RC} \\
(n=550)\end{array}$ & $\begin{array}{c}\text { ASC } \\
(n=550)\end{array}$ & $\begin{array}{l}\text { COMP } \\
(n=550)\end{array}$ & $\begin{array}{c}\mathrm{RC} \\
(n=560)\end{array}$ & $\begin{array}{c}\mathrm{ASC} \\
(n=553) \\
\end{array}$ & $\begin{array}{l}\text { COMP } \\
(n=561) \\
\end{array}$ \\
\hline $\begin{array}{l}1 \text { - L'intervalle inter-génésique doit être supérieur } \\
\text { ou égal à } 24 \text { mois } \\
2 \text { - Les femmes qui ont des grossesses rapprochées } \\
\text { doivent utiliser une méthode de PF } \\
3 \text { - Une femme non mariée doit utiliser une } \\
\text { méthode contraceptive pour éviter une } \\
\text { grossesse } \\
4 \text { - Une femme peut décider seule d'appliquer une } \\
\text { méthode d'espacement des } \\
\text { naissances si son mari ne se soucie pas de sa } \\
\text { santé } \\
5 \text { - La pilule peut être employée à n'importe quel } \\
\text { âge depuis l'adolescence jusqu'à la } \\
\text { ménopause } \\
6 \text { - Approuve l'utilisation de la pilule par une } \\
\text { femme qu'elle ait un enfant ou non } \\
7 \text { - On peut se servir du préservatif en parallèle avec } \\
\text { d'autres méthodes de PF pour la } \\
\text { prévention des IST/VIH/SIDA et pour obtenir } \\
\text { une protection supplémentaire } \\
\text { contre la grossesse }\end{array}$ & $\begin{array}{l}0.78 \\
0.77 \\
0.30\end{array}$ & $\begin{array}{l}0.66 \\
0.65 \\
0.29\end{array}$ & 0.92 & $\begin{array}{l}0.97^{*} \\
0.91^{*} \\
0.40^{*}\end{array}$ & $0.98^{*}$ & $\begin{array}{l}0.98^{*} \\
0.95 \\
0.48\end{array}$ \\
\hline $\begin{array}{l}\text { Indice calculé sur les } 7 \text { items } \\
\text { Variance } \\
\text { Indice calculé sur une échelle de } 10 \text { points possibles }\end{array}$ & $\begin{array}{l}3.38 \\
4.72 \\
4.83 \\
\end{array}$ & $\begin{array}{l}3.16 \\
6.303 \\
\mathbf{4 . 5 1} \\
\end{array}$ & $\begin{array}{c}4.67 \\
3.112 \\
6.67 \\
\end{array}$ & $\begin{array}{r}4.32 \\
2.567 \\
6.17 * \\
\end{array}$ & $\begin{array}{l}4.04 \\
2.484 \\
5.77 *\end{array}$ & $\begin{array}{r}4.33 \\
2.302 \\
6.19 \\
\end{array}$ \\
\hline
\end{tabular}

Sources : CAP femmes pré-intervention (2001) et post-intervention (2003).

$* \mathrm{p}<.05$ 
Tableau 14 : Indice synthétique des attitudes vis-à-vis du VIH/SIDA

\begin{tabular}{|c|c|c|c|c|c|c|}
\hline \multirow[b]{2}{*}{ Indicateurs } & \multicolumn{3}{|c|}{ Pré-intervention } & \multicolumn{3}{|c|}{ Post-intervention } \\
\hline & $\begin{array}{c}\mathrm{RC} \\
(n=550)\end{array}$ & $\begin{array}{c}\mathrm{ASC} \\
(n=550)\end{array}$ & $\begin{array}{l}\text { COMP } \\
(n=550) \\
\end{array}$ & $\begin{array}{c}\mathrm{RC} \\
(n=560)\end{array}$ & $\begin{array}{c}\mathrm{ASC} \\
(n=553) \\
\end{array}$ & $\begin{array}{l}\text { COMP } \\
(n=561) \\
\end{array}$ \\
\hline $\begin{array}{l}1 \text { - Ont déjà discuté des moyens de protection contre le VIH/SIDA avec le mari/partenaire } \\
2 \text { - Pensent qu'il est acceptable de discuter des problèmes de SIDA avec les relais communautaires } \\
3 \text { - Pensent qu'il est acceptable de discuter des problèmes de SIDA dans les cases de santé } \\
4 \text { - Pensent qu'il est acceptable de discuter des problèmes de SIDA dans les postes de santé } \\
5 \text { - Pensent qu'il est acceptable de discuter des problèmes de SIDA à la radio } \\
6 \text { - Pensent qu'il est acceptable de discuter des problèmes de SIDA à la télévision }\end{array}$ & $\begin{array}{l}0.17 \\
0.79 \\
0.79 \\
0.80 \\
0.84 \\
0.81\end{array}$ & $\begin{array}{l}0.18 \\
0.85 \\
0.85 \\
0.85 \\
0.85 \\
0.84\end{array}$ & $\begin{array}{l}0.24 \\
0.97 \\
0.97 \\
0.97 \\
0.97 \\
0.97\end{array}$ & $\begin{array}{c}0.19 \\
0.97 * \\
0.98^{*} \\
0.98^{*} \\
0.98^{*} \\
0.97^{*}\end{array}$ & $\begin{array}{l}0.28^{*} \\
0.97 * \\
0.97 * \\
0.97 * \\
0.97^{*} \\
0.97 *\end{array}$ & $\begin{array}{c}0.27 \\
0.99 \\
0.99 \\
1^{*} \\
1^{*} \\
0.98\end{array}$ \\
\hline $\begin{array}{l}\text { Indice calculé sur les } 8 \text { items } \\
\text { Variance } \\
\text { Indice calculé sur une échelle de } 10 \text { points possibles }\end{array}$ & $\begin{array}{c}4.19 \\
3.890 \\
6.98 \\
\end{array}$ & $\begin{array}{c}4.41 \\
3.430 \\
7.35 \\
\end{array}$ & $\begin{array}{c}\mathbf{5 . 0 9} \\
0.936 \\
\mathbf{8 . 4 8} \\
\end{array}$ & \begin{tabular}{|c|}
$\mathbf{5 . 0 6}$ \\
0.775 \\
$\mathbf{8 . 4 3 *}$ \\
\end{tabular} & $\begin{array}{c}5.14 \\
0.907 \\
8.57 * \\
\end{array}$ & $\begin{array}{c}5.23 \\
0.349 \\
\mathbf{8 . 7 2} \\
\end{array}$ \\
\hline
\end{tabular}

Sources : CAP femmes pré-intervention (2001) et post-intervention (2003).

$* \mathrm{p}<.05$ 
Tableau 15 : Femmes qui ne sont pas sous méthode et qui n'ont pas l'intention d'en utiliser dans le future selon la raison et selon la zone

\begin{tabular}{lccc}
\hline \hline Raisons & Zone RC & Zone ASC & $\begin{array}{c}\text { Zone de } \\
\text { comparaison }\end{array}$ \\
\hline 1. Veut des enfants & $(\%)$ & $\mathbf{( \% )}$ & $\mathbf{( \% )}$ \\
2. Opposée à la PF & 24 & 20 & 27 \\
3. Problèmes de santé & 13 & 22 & 10 \\
4. Effets secondaires & 2 & 8 & 18 \\
5. Ménopause & 3 & 2 & 23 \\
6. Pas commode & 2 & 2 & 3 \\
\end{tabular}

Source : Enquête CAP Femmes Post-test 